\title{
A shared cortical bottleneck underlying Attentional Blink and Psychological Refractory Period
}

\author{
Sébastien Marti a,b,*, Mariano Sigman ${ }^{\mathrm{e}}$, Stanislas Dehaene ${ }^{\mathrm{a}, \mathrm{b}, \mathrm{c}, \mathrm{d}}$ \\ a INSERM, U992, Cognitive Neuroimaging Unit, F-91191 Gif/Yvette, France \\ b CEA, DSV/I2BM, NeuroSpin Center, F-91191 Gif/Yvette, France \\ c Collège de France, F-75005 Paris, France \\ d University Paris 11, Orsay, France \\ e Integrative Neuroscience Laboratory, Physics Department, University of Buenos Aires, Argentina
}

\section{A R T I C L E I N F O}

\section{Article history:}

Received 20 July 2011

Revised 22 September 2011

Accepted 23 September 2011

Available online $\mathrm{xxxx}$

\section{Keywords:}

Psychological refractory period

Attentional blink

Dual-task

Attention

Consciousness

Magnetoencephalography

\begin{abstract}
A B S T R A C T
Doing two things at once is difficult. When two tasks have to be performed within a short interval, the second is sharply delayed, an effect called the Psychological Refractory Period (PRP). Similarly, when two successive visual targets are briefly flashed, people may fail to detect the second target (Attentional Blink or $A B$ ). Although AB and PRP are typically studied in very different paradigms, a recent detailed neuromimetic model suggests that both might arise from the same serial stage during which stimuli gain access to consciousness and, as a result, can be arbitrarily routed to any other appropriate processor. Here, in agreement with this model, we demonstrate that $A B$ and PRP can be obtained on alternate trials of the same cross-modal paradigm and result from limitations in the same brain mechanisms. We asked participants to respond as fast as possible to an auditory target $\mathrm{T} 1$ and then to a visual target $\mathrm{T} 2$ embedded in a series of distractors, while brain activity was recorded with magneto-encephalography (MEG). For identical stimuli, we observed a mixture of blinked trials, where T2 was entirely missed, and PRP trials, where T2 processing was delayed. MEG recordings showed that PRP and blinked trials underwent identical sensory processing in visual occipito-temporal cortices, even including the non-conscious separation of targets from distractors. However, late activations in frontal cortex (>350 ms), strongly influenced by the speed of task-1 execution, were delayed in PRP trials and absent in blinked trials. Our findings suggest that PRP and AB arise from similar cortical stages, can occur with the same exact stimuli, and are merely distinguished by trial-by-trial fluctuations in task processing.
\end{abstract}

(c) 2011 Elsevier Inc. All rights reserved.

\section{Introduction}

Despite a highly parallel anatomical wiring, the human brain has fundamental limitations when multiple tasks have to be performed in close succession. For example, speaking on the phone alters driving performance and vice versa (Becic et al., 2010; Levy et al., 2006). Recent studies on dual tasks suggest that multiple stimuli can be processed in parallel at a sensory level, but that conscious access and/or response selection to these stimuli are strictly serial (Marti et al., 2010; Pashler, 1994; Pashler and Johnston, 1989; Sigman and Dehaene, 2005, 2006, 2008). Here, our goal was to explore the temporal sequence of brain events leading to conscious access in a dual-task situation and, specifically, to examine how brain modules which may operate in parallel interact via a routing mechanism which poses a bottleneck reflecting serial mechanisms of conscious perception.

\footnotetext{
* Corresponding author at: Cognitive Neuroimaging Unit - Inserm U.992, CEA/DSV/I2BM/ NeuroSpin, Bât 145, Point Courrier 156, F-91191 GIF/YVETTE, France. Fax: + 33169087973. E-mail address: sebastien.marti@cea.fr (S. Marti).
}

Only a few hundred milliseconds are needed for people to press a button according to the nature of a stimulus. But if they have to perform a similar task with a second stimulus presented simultaneously or in close temporal proximity, their second response time will be much slower, a phenomenon called the Psychological Refractory Period (PRP) (Pashler, 1994). Classical theoretical models of the PRP propose that tasks can be divided into three consecutive stages with distinct relations to the serial/parallel divide: perception, central decision, and motor response. Sensory encoding of the stimulus occurs in the first stage. It is followed by a strictly serial central decision, linking sensory information to arbitrary motor action. The motor stage is the implementation of the motor response (Pashler, 1994; Pashler and Johnston, 1989). More recently, the central interference model was refined to suggest that the central stage accumulates noisy sensory evidence towards a decision threshold. When this threshold is reached, a motor response is emitted (Gold and Shadlen, 2001; Sigman and Dehaene, 2005, 2006, 2008; Zylberberg et al., 2010). The model assumes that while the sensory and motor stages can be performed in parallel with another task, the central decision stage is strictly serial and constitutes a bottleneck in the processing of the two tasks. In other words, both perception and motor 
execution are unaffected by dual-task interference, but only the central decision is delayed during the PRP.

Evidence in support of this scheme initially came from behavioural studies evidencing a dissociated impact on response times of experimental factors affecting the perceptual, central and motor stages (Sigman and Dehaene, 2005). Time-resolved neuroimaging studies with event-related potentials (ERP) confirmed that the latencies of sensory components such as the N1 and P1 are unaffected by the PRP effect, although their amplitude can be attenuated (Brisson and Jolicoeur, 2007a,b; Sigman and Dehaene, 2008). On the other hand, at the central level, the amplitude of later components such as the P3b is unaffected by the PRP, but their latency is strongly shifted in time, compatible with serial postponement (Dell'acqua et al., 2005; Sigman and Dehaene, 2008). Other studies using functional magnetic resonance imaging (fMRI) have shed some light on the brain areas involved in the PRP effect (Dux et al., 2006; Sigman and Dehaene, 2008). Using time resolved fMRI, Sigman and Dehaene (2008), showed that at least part of the perceptual processing of the second target can be achieved in parallel to task 1, but that activations in parietal and frontal cortex related to decision making are strictly serial.

A recent neuronal implementation of this model (Zylberberg et al., 2010), which successfully accounted for a wide variety of results in the dual-task literature leads to refined predictions about the neurophysiological mechanisms of the PRP. In this model, the sensory integration of the second target is achieved via successive sets of neurons with receptive fields of increased complexity. At the top of this sensory hierarchy, recurrent connections between the neuronal layers insure a slow exponential decay of sensory information, resulting in a form of sensory memory or buffer. Hence, the availability of an active representation in this sensory buffer defines a time period during which sensory information is accessible to further processing. The buffer allows information to wait for access to a central capacitylimited "router" system, consisting of neurons capable of flexibly interconnecting sensory categories with response intentions. Subsets of router neurons specific to stimulus-response pairs are selected via task-setting neurons. Once selected, router neurons are able to accumulate sensory evidence until a subset of them reach a threshold and trigger motor neurons coding for the response. These motor neurons then send back inhibitory signals to sensory, router and task-setting neurons which terminate the processing of the task. Thus, the model includes a detailed implementation, with realistic spiking neurons, of the distinction between parallel sensory integration and serial central processing.

A key property of the central interference model, which is submitted here to experimental scrutiny, states that if T1 central processing exceeds the duration of the decaying $\mathrm{T} 2$ representation in the sensory buffer, then T2 sensory information can no further be retrieved. In such situation, participants would not be able to consciously report the second target, nor to perform the second task: they would simply report a subjective absence of $\mathrm{T} 2$. In fact, this property fits precisely with another well known dual-task limitation: the attentional blink (AB) (Raymond et al., 1992). The classical experimental observation of AB (Chun and Potter, 1995; Raymond et al., 1992) consists in asking participants to attend to a stream of successive visual stimuli and, at the end, report the identity of occasional targets (e.g. numbers in a stream of letters). Whenever two targets occur in close succession, within approximately half a second, there is a high probability that the second target will be missed (Raymond et al., 1992), except if they immediately follow each other ("lag 1 sparing") (Potter et al., 1998). Since this first pioneering observation, the inability to detect or report a second target presented within a narrow time window after a first target, which we and others consider as definitional of the attentional blink (Kawahara et al., 2003), has been repeatedly observed in a broad variety of visual, auditory, and crossmodal paradigms (Arnell and Larson, 2002; Duncan et al., 1994; Jolicoeur, 1999b, d; Raymond et al., 1992; Tremblay et al., 2005).
The central interference model share some aspects with previous bottleneck models of the AB (Chun and Potter, 1995), but it makes the specific proposal that $\mathrm{AB}$ and PRP arise from the same central processing stage, at the end of central $\mathrm{T} 1$ processing, when participants attempt to recover T2 from the buffer. The only difference is that retrieval is successful on PRP trials, and fails on AB trials. Experimentally, previous studies have indeed revealed several similarities between $A B$ and PRP. First, both effects are observed when two target items are separated by less than $\sim 500 \mathrm{~ms}$. Second, as predicted by bottleneck models, both the PRP and the AB are affected by the speed of T1 processing (Jolicoeur, 1999a,b,c,d). Third, at the brain level, event-related potentials (ERPs) studies reveal that early sensory components are preserved during $A B$ and PRP alike, and their latency is not affected by the inter target lag (Sergent et al., 2005; Sergent and Dehaene, 2004). Fourth, the P3 component is delayed when the second target is detected, as in the PRP (Ptito et al., 2008; Sergent et al., 2005; Vogel and Luck, 2002), and completely vanishes when the target is missed or blinked (Sergent et al., 2005; Sergent and Dehaene, 2004; Sigman and Dehaene, 2006).

Nevertheless, these parallels between $A B$ and PRP result from independent experiments using different tasks, participants and even laboratories, and hence they do not constitute a proof that the $A B$ and the PRP are related phenomena sharing similar brain mechanisms. Furthermore, empirically, the two paradigms differ in several ways. One such difference is lag 1 sparing: in $A B$, when $\mathrm{T} 1$ and $\mathrm{T} 2$ are presented in immediate succession, perception of T2 is usually quite good while in the PRP, such a short lag leads to the slowest responses to T2. Another difference involves crossmodality and task switching: the majority of PRP experiments rely on two distinct successive tasks, usually involving different sensory modalities (to avoid low-level sensory interference), while the majority of $A B$ experiments involve a single visual presentation stream and a single task, typically the unspeeded report of the target stimuli. While there is evidence that an $A B$ can occur cross-modally (Arnell, 2006; Arnell and Jenkins, 2004; Arnell and Larson, 2002; Dell'Acqua et al., 2003; Hein et al., 2006; Ptito et al., 2008) these results have been controversial (Duncan et al., 1997; Martens et al., 2010a). Potter et al. (1998) proposed that the deficit observed on T2 in crossmodal paradigms reflected a task-switching effect rather than the AB. However, Arnell \& Larson (2002) showed that, independently of task switching, an $\mathrm{AB}$ along with a lag-1 sparing effect can be observed with an auditory $\mathrm{T} 1$ and a visual T2. In addition, a recent electrophysiological study minimized task-switching demands and showed that, independently of T1 modality, the P3 component related to the perceived second target was delayed (Ptito et al., 2008), which is a typical observation in ERP studies of the AB (Arnell, 2006; Sergent et al., 2005; Vogel et al., 1998). In fact, an AB is even observed with tactile stimuli (Dell'Acqua et al., 2001; Hillstrom et al., 2002). Hence, even if the topic is still debated, these results support the existence of a crossmodal AB.

The best evidence to date that $A B$ and PRP may share common mechanisms comes from behavioural experiments showing that, both within and across modalities, slow response times to T1 are associated with a larger AB compared to fast response times (Jolicoeur, 1999a,b,c,d; Jolicoeur et al., 2000). This shows that the duration of task 1 , which is the main determinant of the PRP, also influences the size of the $A B$. From these results, it has been suggested that both $A B$ and PRP arise from an amodal central bottleneck which would delay attention allocation to T2 (the PRP) and would eventually prevent its shortterm consolidation (the AB) (Jolicoeur, 1999a; Jolicoeur et al., 2000).

In this context, the goal of the present experiment was to further test the hypothesis that $\mathrm{AB}$ and $\mathrm{PRP}$ result from common brain mechanisms and can be obtained within a single experiment. Specifically, we tested the predictions that (1) an AB should be easily obtained in a typical cross-modal PRP situation. (2) RT1 should influence both the PRP and the size of the AB. (3) At the brain level, activations 
in the sensory cortices should be similar for both PRP and blinked trials and time-locked to the onset of T2; however, activations in frontal, parietal and anterior cingulate cortices should be present in PRP trials but not in blinked trials. (4) During the PRP, these central activations should be influenced by RT1 but not by the inter-target lag.

\section{Method}

\section{Subjects}

Twenty-two subjects participated to the experiment (12 women) aged between 20 and 35 years old. Informed consent was obtained before testing, and subjects received a compensation of $120 €$. All subjects were naïve with respect to the task and all had normal or corrected to normal vision. Four subjects were discarded because of technical difficulties during the recording. The behavioral results of the 18 remaining subjects are described in the results section and detailed in the supplementary materials. All subjects showed a PRP effect but six had less than $10 \%$ of blinked trials at lag 1 . Since one of our main goals was to compare signals in seen versus blinked trials within the same subjects, we only considered for subsequent MEG analysis the 12 participants showing a significant blink effect. In the remaining group, two subjects were excluded because of an abnormal high level of noise in the MEG signal. Thus, in the end, ten subjects were included in the MEG analyses.

\section{Stimuli and apparatus}

All participants performed a dual-task in which the first target was a monotonic sound presented to both ears. The target sound could be a high pitch $(1100 \mathrm{~Hz})$ or a low pitch $(1000 \mathrm{~Hz})$ and was presented for $84 \mathrm{~ms}$. The second target was a black letter $\left(0.64^{\circ}\right)$, either the letter "Y" or the letter "Z", presented on a white background. The target letter was embedded in a visual stream of 12 random black letters used as distractors. Each letter was presented at the centre of the screen for $34 \mathrm{~ms}$ with an inter stimulus interval of $66 \mathrm{~ms}$. The target sound was always synchronized to the third distractor and followed by the second target after a variable inter target lag: $100,200,400$ or $900 \mathrm{~ms}$. In a fifth condition, T2 was replaced by a distractor (Distractor condition). Participants were instructed (1) to respond as fast as possible first to the sound and then to the letter, (2) to respond as soon as the corresponding stimulus appeared, thus avoiding "grouped responses", (3) that the second stimulus would occasionally be absent, in which case they should simply not perform the second task. As in a previous study (Wong, 2002), T2-present trials that failed to be responded were classified as "blinked", and the rest as "seen". In all analyses, we only considered trials with a correct T1 response and, for PRP analysis, a correct $\mathrm{T} 2$ response.

Trials began with the word "GO" presented centrally for $500 \mathrm{~ms}$. A fixation cross then appeared immediately (duration: $1000 \mathrm{~ms}$ ) followed by the first letter of the rapid visual stream. After the 13 letters of the RSVP, a blank screen was presented for $3000 \mathrm{~ms}$ before the beginning of the next trial.

The experiment consisted of two training blocks of 20 trials each, one to practice the auditory task and the other one to practice the visual task, followed by 5 experimental blocks. In four of these experimental blocks, participants performed 100 trials of the dual-task and in one block they performed 50 trials of only the visual task while they had to listen passively to the sound (T1 irrelevant condition). Thus, a maximum of 80 trials by inter target lag were recorded. Trials with reaction times inferior to $300 \mathrm{~ms}$, superior to $2000 \mathrm{~ms}$ for T1, or superior to $2500 \mathrm{~ms}$ for T2 were excluded ( $2.1 \pm 2.5 \%$ of trials rejected). The order of the experimental blocks was counter-balanced across subjects. Both training and experimental blocks were performed while the subjects sat back in the MEG chair so that training and experimental contexts were identical.
Stimuli were back projected (refresh rate: $60 \mathrm{~Hz}$ ) on a screen placed $60 \mathrm{~cm}$ in front of the subject under standard overhead fluorescent lighting. The sequence was controlled by a Pentium IV PC running E-Prime 1.1 software (PST Inc.). Sounds were presented through nonmagnetic earphones. The sound intensity was constant across subjects and set to be comfortable. None of the subjects reported any problem hearing the sounds and all performed well the auditory task. We used a five button non-magnetic response box (Cambridge Research Systems Ltd., Fibre Optic Response Pad) to record their motor responses. Six of the subjects used their left hand to respond to the sound (middle finger for low pitch, index for high pitch) and their right hand to respond to the letter (index for the letter "Y" and middle finger for the letter "Z"). Four subjects used their right hand to respond to the sound (index for low pitch, middle finger for high pitch) and their left hand to respond to the letter (middle finger for "Y" and index for "Z").

\section{MEG recordings}

While subjects performed the cognitive tasks, we continuously recorded brain activity using a 306-channel whole-head magnetometer (Elekta Neuromag®) inside a magnetically shielded room (Maxshield) to decrease electromagnetic noise. Channels were organized in 102 triplets, each one composed of a magnetometer and two orthogonal planar gradiometers. MEG signals were continuously recorded at a sampling rate of $1000 \mathrm{~Hz}$. Four head position indicators were placed over frontal and mastoïdian skull areas. The subject's head position was then measured at the beginning of each run using an isotrak polhemus Inc. system to compensate for head movements. Horizontal and vertical electro-oculograms and electrocardiogram were recorded simultaneously for offline rejection of eye movements and cardiac artefacts.

\section{Data preprocessing}

Signal Space Separation (SSS) method was applied to decrease the impact of external noise and sensor artefacts by separating the magnetic fields arising from sources inside the sensor helmet and those arising from sources outside (Taulu et al., 2004). MEG signals were lowpass filtered at $330 \mathrm{~Hz}$. Gradiometers and magnetometers with amplitudes continuously exceeding $3000 \mathrm{fT} / \mathrm{cm}^{2}$ and $3000 \mathrm{fT}$ respectively were set as bad channels and excluded from further analysis (range of bad channels: 1 to 6 across subjects). SSS correction, head movement compensation and bad channels correction were applied using the MaxFilter Software (Elekta Neuromag $®$ ). Continuous data were then epoched using Fieldtrip software (http://fieldtrip.fcdonders.nl/). Trials were time locked to the onset of $\mathrm{T} 1$ with a time window starting $500 \mathrm{~ms}$ before T1 onset (i.e. $300 \mathrm{~ms}$ before the beginning of the RSVP) and ending $2000 \mathrm{~ms}$ after. A baseline correction was applied for each trial using the first $200 \mathrm{~ms}$ of the epoch. The variance of the MEG signals across sensors was computed for each trial and displayed in a scatter plot. This variance was used as an index to visually inspect trials that might be artefacted by muscles or movement. After visual inspection, bad trials were rejected (the proportion of rejected trials across subjects varied from 2 to $8.75 \%$ ). Independent component analyses were applied separately for each type of sensor. To identify the components related to the cardiac artefact and to the eye movement, we computed correlations between each component and the ECG, and between each component and the EOG and visually inspected their topography. Once identified, these components were subtracted out from the raw data.

\section{Statistical analyses}

To examine differences between experimental conditions, we performed paired t-tests with a threshold set at $\mathrm{p}=0.05$ after applying a low pass filter of $30 \mathrm{~Hz}$. A correction for multiple comparisons was then applied using cluster-based permutations tests, with a final corrected-level threshold set at $\mathrm{p}=0.05$. On average, 13 sensors were 
included in a cluster with a minimum of 2 channels. The analyses were performed over a $40 \mathrm{~ms}$ time window centered on the peak of each component. Given the different nature of the three types of sensors, the statistical analyses were performed separately for longitudinal gradiometers, latitudinal gradiometers and magnetometers.

\section{Multiple regression analyses}

To probe the time course of specific brain components, we used a multiple-regression analysis (Sigman and Dehaene, 2008) whereby templates of brain activity identified in the Lag-9 condition were used as topographic multiple regressors for brain activity in other conditions and at other time points. First, we averaged the ERFs for the lag 9 condition across subjects and computed the sum of squares across sensors in order to identify the components specific to the presentation of each target. This measure resulted in a sequence of easily distinguishable peaks. We then compared the Lag 9 condition to the relevant control conditions ( $\mathrm{T} 1$ irrelevant and Distractor conditions respectively for $\mathrm{T} 1$ and $\mathrm{T} 2$ ) using cluster-based permutation tests (see Statistical analyses section). Each peak identified and corresponding to a significant difference between the Lag 9 condition and the relevant control condition was defined as a component. Once this procedure was done on the group average, we used it as a template and repeated the same procedure for each subject. We computed the sum of squares across sensors and subtracted the Lag 9 condition to the relevant control condition. Non-filtered data were then averaged over a time window of $50 \mathrm{~ms}$ around the peak of each component. As detailed below, this procedure resulted in two sets of four components (one for each task) for each subject. The topographies of these components were then used as regressors in a multiple regression which modelled the measured topography of each of the other lag conditions (i.e. Lag 1, 2 and 4) at each time point. We report here the beta values of the regression for each time point of a time window starting $500 \mathrm{~ms}$ before the presentation of T1 and ending $2000 \mathrm{~ms}$ after. For brevity we refer to this curve as the time course of a component. This method resulted in a single time course for each magnetic component identified and gave us information about both its timing and its amplitude. In addition, two parameters were measured on the time courses obtained with the multiple regressions: the peak latency and the width of each component. For each Lag condition and for each component, we selected a time window around the maximum of the time-course of each component ( $300 \mathrm{~ms}$, for the M270 and M350 components, $400 \mathrm{~ms}$ for the M430, and $600 \mathrm{~ms}$ for the M550). To measure the latency while avoiding typical numerical instabilities in the computation of the peak, we determined a broad peak considering all time points for which the beta values exceeded the 75th percentile of the distribution. This robust estimation of the peak is non-parametric (i.e. does not assume a specific shape of the peak). We measured the latency as the median of the time points exceeding the $75 \%$ percentile and the width of the component as the time interval covered by these time points.

\section{Anatomical MRI}

Anatomical magnetic resonance images (MRI) were obtained for each participant after the MEG experiment with a 3-T Siemens MRI scanner, with a resolution of $1 \times 1 \times 1.1 \mathrm{~mm}$. The head position indicator and the digitized head shape were used for the co-registration of the anatomical images with the MEG signals. The grey and white matters of the MRI were then segmented using BrainVisa / Anatomist software package (http://brainvisa.info/).

\section{Source localisations of the MEG signals}

The head and cortical surfaces were reconstructed for each subject using BrainStorm software (http://neuroimage.usc.edu/brainstorm/). Models of the cortex and of the head were used to estimate the current-source density distribution over the cortical surface. The forward modelling was computed using an overlapping-spheres analytical model. The inverse modelling was based on minimum norm solutions (weighted minimum-norm current estimate, wMNE). For each subject, the sources were projected to a standard anatomical template (MNI) and then transformed in $\mathrm{Z}$ scores relative to the baseline. The absolute values of the $Z$ scores were then averaged across subjects. For presentation purposes, the sources were spatially smoothed over 5 neighboured vertices.

\section{Results}

\section{Behavioural results}

\section{The psychological refractory period}

Fig. 1B represents the mean reaction times across subjects for tasks 1 (RT1) and 2 (RT2) as a function of the inter-target lag. The central interference model proposes that the response to task 2 is delayed until $\mathrm{T} 1$ central processing is complete. Our data fit this by-now classical prediction of the PRP. We found a significant effect of inter-target lag on RT2 $(\mathrm{F}(3,27)=31.70, \mathrm{p}<0.001)$ but not on RT1, which shows that RT2 was significantly slower when the inter-target lag decreased while RT1 remained unaffected. The slope was $-1.03 \pm 0.12$ between lag 1 and 2 and closer to 0 as the lag increased $(-0.40 \pm 0.07$ between lag 2 and 3 , and $-0.13 \pm 0.05$ between lag 3 and 4 ). This shows that during the wait period, decreasing the inter-target lag increased RT2 correspondingly. The mean correlation between RT1 and RT2 was strong at short lag (mean Pearson $r=0.62 \pm 0.05$ ) and became progressively weaker as the lag increased (lag 2: $0.46 \pm 0.07$; lag 4: $0.40 \pm 0.07$; lag 9: $0.18 \pm 0.07)$. This means that, at short lags, a large part of the variance of RT2 was due to the variable completion of task 1 .

\section{The attentional blink}

Since the existence of a robust cross-modal blink is debated, we first verified if we were capable of inducing, under our experimental conditions, a significant $\mathrm{AB}$ effect. We computed, within trials with a correct response to $\mathrm{T} 1$, the proportion of correct $\mathrm{T} 2$ responses for each inter-target lag and found that this proportion decreased when the lag decreased $(F(3,27)=19.09, p<0.001$; Fig. $1 C)$, revealing a significant $A B$ effect in our paradigm. Second, we examined the proportion of blinked trials as a function of RT1 speed (Fig. 1D). According to the bottleneck model and in agreement with previous observations (Jolicoeur, 1999a,b,d), this proportion should increase for slow RT1 compared to fast RT1. For each subject, we split the trials into those below or above the median RT1, and we computed a repeated measure ANOVA on the proportion of blinked trials with slow/fast RT1 and inter-target lag as within-subject factors. The results revealed an effect of $\operatorname{Lag}(F(3,27)=15.82, \mathrm{p}<0.001)$ and of RT1 speed $(F(3,27)=53.16$, $\mathrm{p}<0.001)$ and, crucially, a significant interaction Lag x RT1 speed ( $F$ $(3,27)=3.18, p=0.04)$. The proportion of blinked trials was higher for slow RT1 compared to fast RT1 for Lag $1(\mathrm{~F}(1,9)=18.06, \mathrm{p}<0.01)$, Lag $2(\mathrm{~F}(1,9)=35.02, \mathrm{p}<0.001)$ and $\operatorname{Lag} 4(\mathrm{~F}(1,9)=25.32, \mathrm{p}<0.001$ but not for Lag 9 (see Fig. 1D). In summary, the duration of task 1 has a strong influence on both the PRP and the size of the AB at short lag intervals.

As detailed in the Method section, eight subjects with valid behavioral data had to be excluded from MEG analyses. Results from the group of 18 participants and those from the 10 participants included in the MEG analysis were comparable, as can be seen in Fig. S1. We again found a significant effect of inter target lag on RT2 $(F(3,51)=40.60, \mathrm{p}<0.001)$ but not on RT1 ( $p=0.2)$, i.e. a strong PRP effect. The proportion of correct T2 trials, given a correct T1 response, again decreased when the lag decreased $(F(3,51)=7.69, p<0.001$; Fig. S1B), revealing a significant AB effect. Finally, an ANOVA with RT1 speed again revealed significant effects of Lag $(F(3,51)=9.63, p<0.001)$, RT1 speed $(F(1,17)=29.44$, $\mathrm{p}<0.001)$ and a significant interaction $(\mathrm{F}(3,51)=3.58, \mathrm{p}<0.05)$. The proportion of blinked trials was higher for slow RT1 compared to fast RT1 for Lag $1(\mathrm{~F}(1,17)=14.92, \mathrm{p}<0.001)$, $\operatorname{Lag} 2(\mathrm{~F}(1,17)=$ 18.00, $\mathrm{p}<0.001)$ and Lag $4(\mathrm{~F}(1,17)=27.80, \mathrm{p}<0.001$ but not 
A) Experimental design

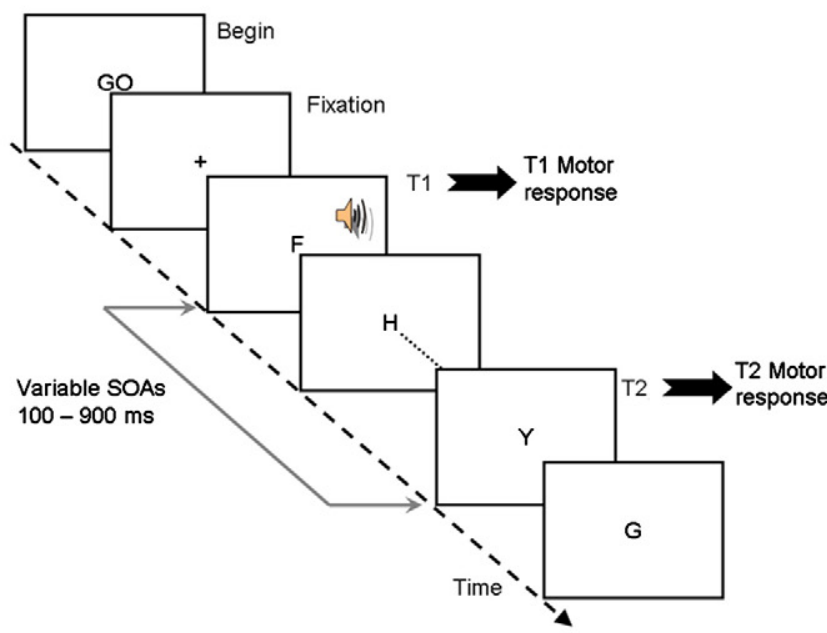

C) The Attentional Blink

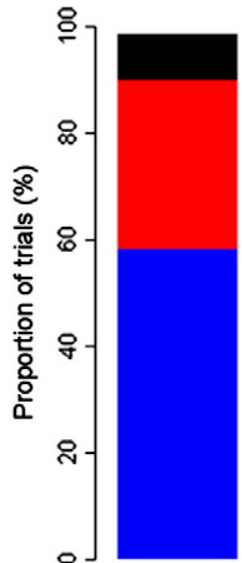

100

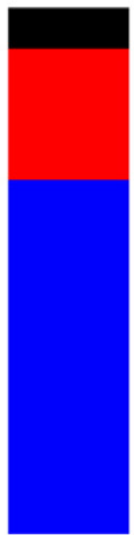

200

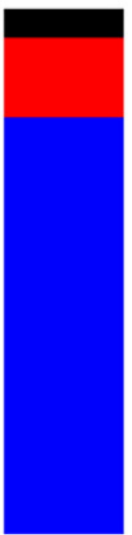

400

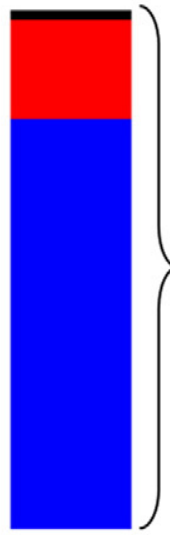

900
Correct T1

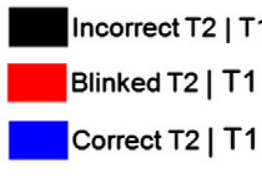

Inter target lag (ms)
B) The Psychological Refractory Period

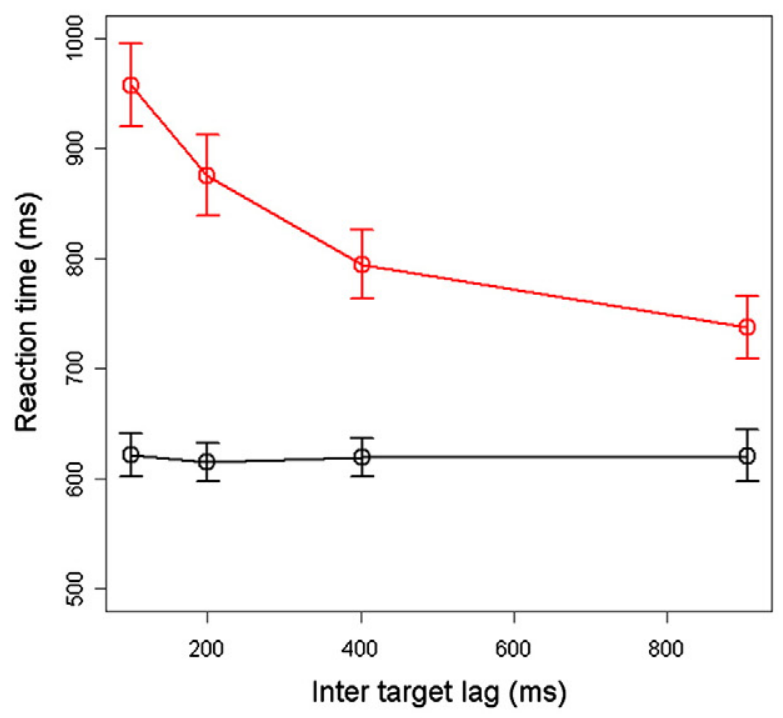

D) Effect of RT1 speed on the AB

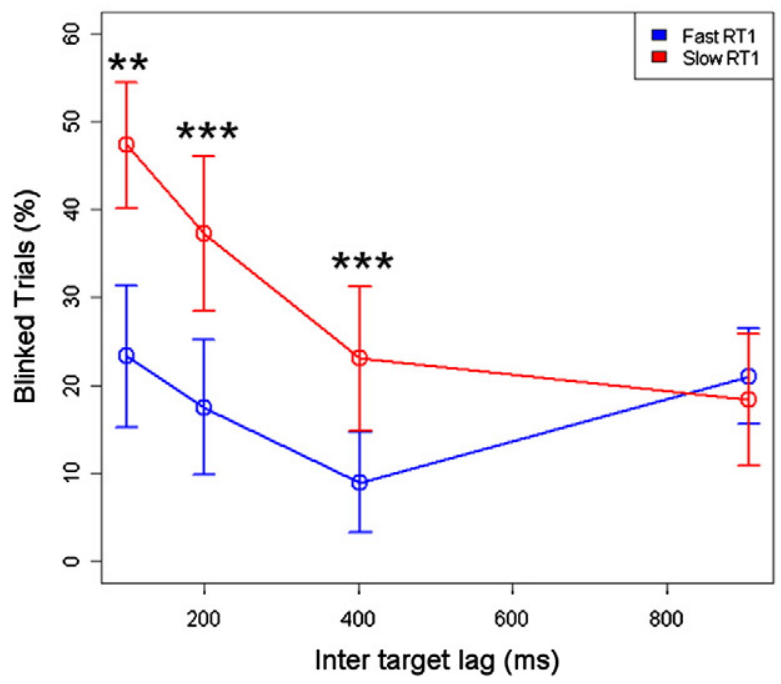

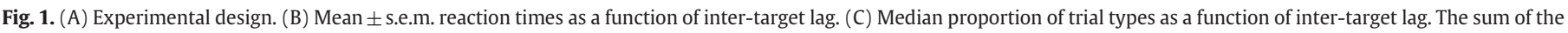

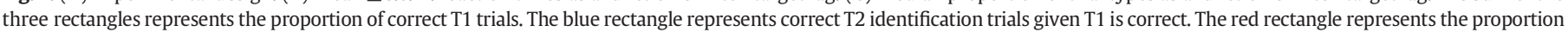

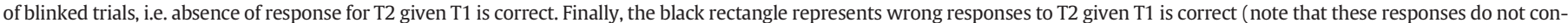

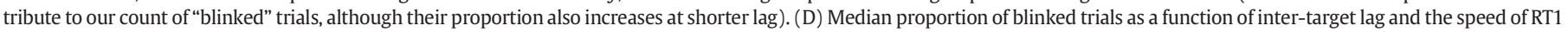
(blue $=$ below median; red $=$ above median ).

significant for Lag $9(\mathrm{p}=0.12)$ (Fig. S1C). In brief, we found exactly the same effects as in the group of 10 subjects included in the MEG analysis, demonstrating that our paradigm produced both a PRP and an AB, and that RT1 speed was a critical factor influencing both phenomena.

\section{Neuroimaging results}

Our general approach to analyse the event-related fields (ERFs) was to use the Lag 9 condition, in which the two tasks can be performed without any interference, to identify a set of evoked components for each task and then to use their topographies as regressors in a multiple linear regression with the other lag conditions where components from both tasks may overlap in time (see the method section for details). Using this approach, we were able to track the dynamics of each of these components in PRP and blinked trials when both tasks interfered.

\section{T1 processing}

Early $\mathrm{T} 1$ perceptual processing is unaffected by task instructions. We were able to identify four components related to T1 processing (Fig. $\mathrm{S} 2$ ). The first of these components peaked around $100 \mathrm{~ms}$ after T1 onset and was therefore named the M100 component. There was no difference in the amplitude of the M100 whether participants responded to $\mathrm{T} 1$ (T1 relevant condition, see the method section) or just listened passively without performing any task 1 (T1 irrelevant), and the latency was comparable in both conditions (Fig. S2A). It suggests that this early sensory stage of processing was not influenced by whether or not task 1 was performed. The sources of the component 
were localized in the superior temporal gyrus and the superior temporal sulcus (Fig. S3A).

Late $T 1$ processing depends on instructions. Three later components were modulated by T1 instructions (M250, M350 and M450). All showed significantly larger amplitudes when $\mathrm{T} 1$ was relevant compared to when T1 was irrelevant (Fig. S2B-D, p $<0.05$, corrected). Source reconstructions revealed that the main generators of the M250 were located in the superior and middle temporal gyri, the left angular and supra marginal gyri and the occipito-parietal cortex (Fig. S3A). By 350 ms the activation in the primary auditory cortex fade-out and we observed a second wave of activation in the occipito-parietal area, the middle temporal gyrus, the angular gyrus and the supra marginal gyrus. The activation then propagated to the left middle and superior frontal gyri around 450-500 ms after T1 onset. This shows that late T1-related activations in frontal and parietal areas were strongly reduced when $\mathrm{T} 1$ was irrelevant.

\section{T2 processing}

Early visual processing is unaffected by stimulus relevance. We examined the effect of stimulus relevance on an occipital component appearing $150 \mathrm{~ms}$ after T2 onset. We did not find any significant difference in the amplitude of this component between the Lag 9 condition and the Distractor-only condition. Thus, this component might be related to the early sensory processing of $\mathrm{T} 2$ which, like the sensory integration of T1, seems to be similar for both target and distractor stimuli.

T2 sensory activations are unaffected by the PRP. The comparison between the Lag 9 condition and the Distractor condition revealed four different components with larger amplitudes for $\mathrm{T} 2(\mathrm{p}<0.05$, corrected; Figs. 2A-D, left part). Examination of their time courses in the lag 1, 2 and 4 conditions where T2 was detected indicated that the M270 and the M350 were time-locked to the onset of T2 and were not affected by the concurrent task 1 (Figs. 2A and B right part, Fig. 6). The effect of the inter-target lag on the peak latencies was significant for both components $(F(2,18)=2350, p<0.001$ and $F$ $(2,18)=419.38, \mathrm{p}<0.001$ respectively). This effect is expected in T2locked components since latencies are measured from the onset of the trial and hence delaying the presentation of $\mathrm{T} 2$ should correspondingly delay the onset of the component.

Source localisation revealed activations in the occipito-temporal area, the middle temporal gyrus, the fusiform gyrus, and the anterior insula (Fig. 3A) in the time range of the M270 (i.e. between 252 and $292 \mathrm{~ms}$ after T2 onset). For the M350, we observed activations in the angular gyrus, the supra-marginal gyrus and the occipito-parietal area. Specifically, time courses of activity in the occipito-temporal and infero-temporal cortices were not affected by the PRP (Fig. 3B), similarly to the pattern observed at the sensor level. In summary, these results show that the M270 and the M350 components are (1) specific to target $\mathrm{T} 2$, as they are evoked by targets relative to distractors, and yet (2) timelocked to T2 onset and therefore unaffected by concurrent T1 processing. These properties indicate that the sensory separation of letter targets from the distractors belongs to the parallel sensory processing stages described by the bottleneck model, prior to central decision, and therefore operate in parallel with $\mathrm{T} 1$ processing.

Central processing of $T 2$ is delayed at short lag. The properties of the M430 and the M550 components were qualitatively and quantitatively different. The effect of the inter-target lag on the peak latency was significant for both components $(\mathrm{F}(2,18)=75.37, \mathrm{p}<0.001$ and $\mathrm{F}(2,18)=$ $12.16, \mathrm{p}<0.001$, Figs. $2 \mathrm{~A}-\mathrm{D}$ right part). Contrast analyses revealed that the peak latency of the M430 was significantly shorter in lag 2 compared to lag $4(\mathrm{~W}=0, \mathrm{p}<0.01)$ but not between lag 2 and lag 1 (Fig. 6A). We found similar results for the M550: the peak latency was shorter in lag 2 compared to lag $4(\mathrm{~W}=1, \mathrm{p}<0.01)$ but not between lag 1 and 2 (Fig. 6A). These findings match precisely the predictions of a central component of the bottleneck model: between lags 2 and 1, accelerating the time of 12 presentation does not accelerate the components, since these components are locked to the completion of T1. Instead, at longer lags, when T1 processing has been completed, accelerating $\mathrm{T} 2$ presentation correspondingly accelerates the peak of the M430 and M550 components.

Source analyses revealed that, for the M430, activations in the right inferior temporal cortex and in the left supra marginal and angular gyri were still present but additional activations were found in the occipito-parietal area, the precuneus and, to a lesser extent, the superior parietal lobule (Fig. 3A). For the M550, the same areas in the parietal lobe were still activated but we observed in addition a massive activation over the frontal cortex, including the precentral gyrus, the superior and middle frontal gyri (mainly in the left hemisphere), the lateral part of the orbito-frontal cortex, and the anterior cingulate cortex (Fig. 3A). The time course revealed that the activity in the superior and middle frontal gyri was delayed during the PRP, which mimicked our observations at the sensor level. This clearly fits our hypothesis of a strictly serial central stage involving frontal cortex as an essential node.

Central processing of $T 2$ is abolished in blinked trials. We next asked whether response components which were delayed during the PRP relative to the onset of T2 also relate to conscious access to T2. Because we observed serial processing and a PRP effect only for the M430 and M550 components, we predicted that these components should also be the only ones to disappear on blinked trials. Indeed, the direct comparison of seen PRP trials to blinked trials at lag 1 revealed significant differences for the M430 and for the M550 but neither for the M270 nor for the M350 (Fig. 4B, p $<0.05$ corrected). The M430 and the M550 were sharply reduced in blinked trials. This shows that the components that were delayed during the PRP were also the ones to vanish when T2 was blinked. The larger amplitude of the MEG signals on seen trials corresponded to activations in the precentral gyrus, the superior and middle frontal gyri, and the occipito-parietal area (Fig. 5A), which is consistent with the activations observed when we compared the lag 9 condition to the Distractor condition (Fig. 3A).

A closer look at the M270 for blinked trials revealed a small but significant difference compared to the Distractor condition on the magnetometers (Fig. 4A, right part). The time courses in Fig. 4B, and the right part of Fig. 5A, show that blinked targets still induced early activations in the supramarginal and angular gyri, and in the occipitotemporal area which were not observed with irrelevant stimuli. Thus, even on blinked trials, the second target was processed at a level deep enough to elicit target-specific activations. However, such activations were not sufficient to trigger the late activations in parietal and frontal areas observed on seen trials (Fig. 5a, left part).

\section{Influence of task 1 duration on $\mathrm{T} 2$ processing}

The central interference model predicts that both PRP and blink effects should be augmented on trials when task 1 responses were slower. To investigate the effect of task-1 duration on T2 processing, we used RT1 as an index of T1 duration and split the seen-T2 trials according to the median of RT1. For each subject, all trials with an RT1 slower than the median were classified as "slow" and trials with RT1 faster than the median were classified as "fast". Our prediction was that only components related to the central processing of T2 should be affected by RT1, while sensory components should remain unaffected. We computed a repeated-measures ANOVA with component type, RT1 speed and inter target lag as within-subject factors. We found significant effects of component $(F(3,27)=945.99$; $\mathrm{p}<0.001)$, RT1 speed $(\mathrm{F}(1,9)=24.32 ; \mathrm{p}<0.001)$, and inter target lag $(\mathrm{F}(2,18)=273.19 ; \mathrm{p}<0.001)$. More importantly, we found a triple interaction Component $x$ RT1 speed $x$ inter target lag $(F(6,54)=2.46$; $\mathrm{p}<0.05)$, revealing an effect of RT1 speed only for late components 


\section{Regression templates: Lag 9 - Distractor}

A) $272 \mathrm{~ms}$

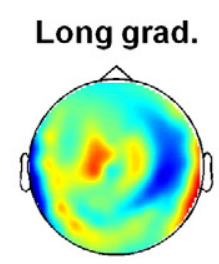

B) $349 \mathrm{~ms}$
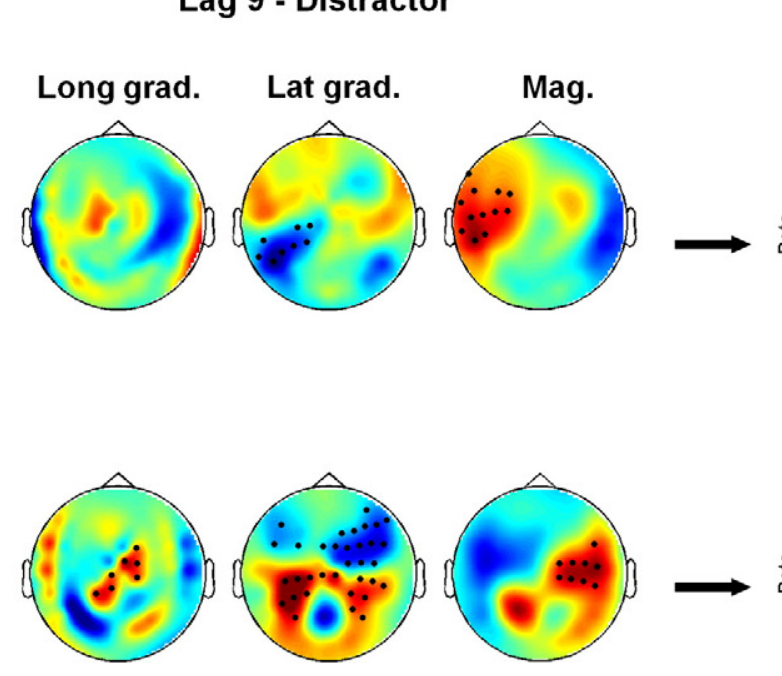

Regressions with Lag 1, 2 and 4
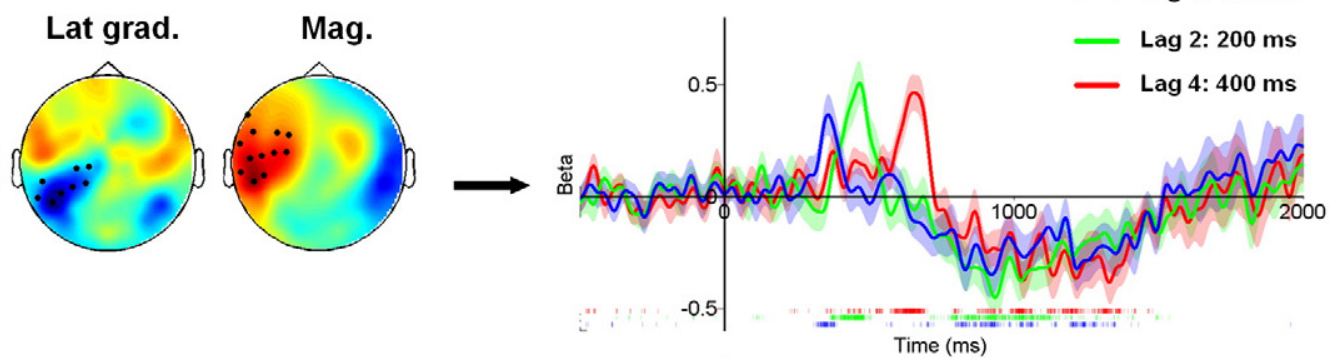

\section{C) $430 \mathrm{~ms}$}
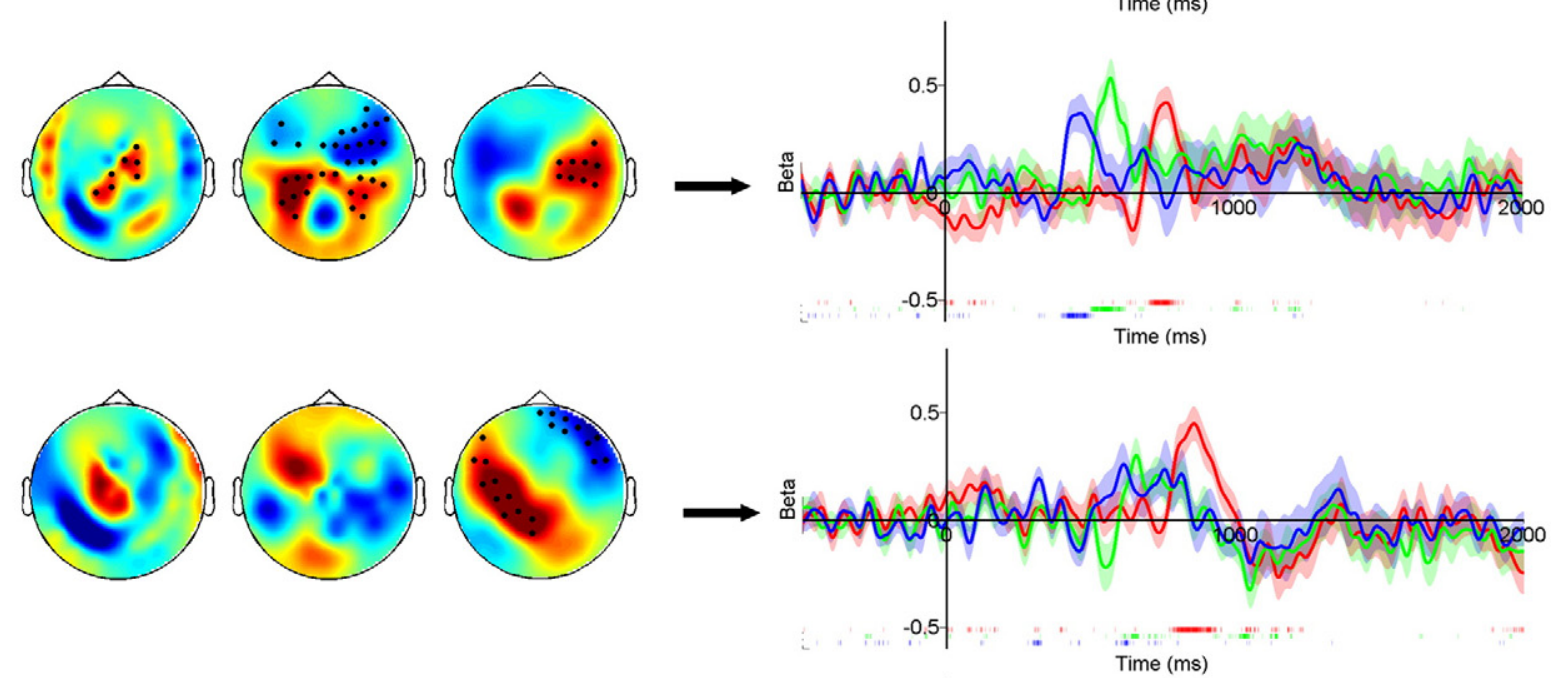

D) $550 \mathrm{~ms}$
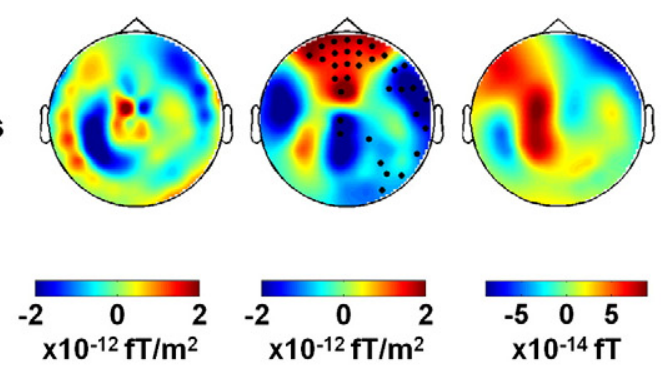

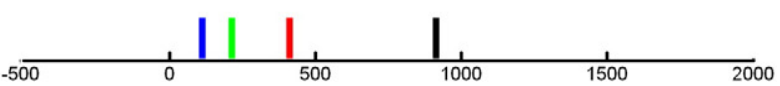

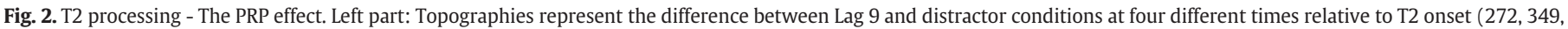

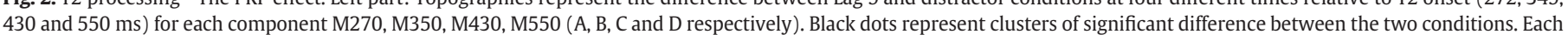

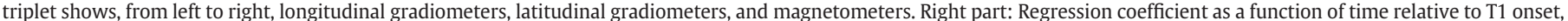

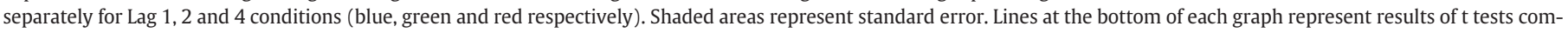

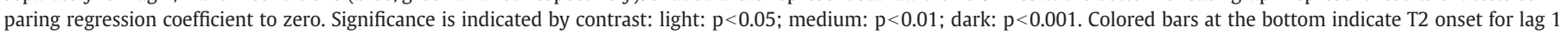
(blue), 2 (green), 4 (red) and 9 (black).

at short lag. Indeed, RT1 speed had no effect at all on the M270 and only a small effect on the peak latency of the M350 (W=3, $\mathrm{p}<0.05)$. On the other hand, a strong effect of RT1 speed was observed on the M430 and on the M550 at lag $1(\mathrm{~W}=0, \mathrm{p}<0.01$ and $\mathrm{W}=0, \mathrm{p}<0.01$ respectively, Fig. $6 \mathrm{~A}$ ). Thus, components showing a strong difference between seen and blinked trials were also influenced by the speed of RT1 and no longer time-locked to T2 onset. These results suggest that, at short lag, task-1 duration mainly influenced T2 central processing while leaving unaffected T2 sensory processing.

Fig. 6B shows the results obtained for the duration of T2 components. As can be seen, we did not find any significant effect either of the inter-target lag or of RT1 speed. The M550 tended to be larger for slow RT1 compared to fast RT1 at lag 1 and 2 but this effect did not reach the threshold for significance. Altogether, our findings show that the timing, but not the duration of T2 components, was sensitive to the experimental factors. For instance, at short lag, the M430 was just pushed back in time by the duration of the T1 task. This absence of effect on the width of the components has theoretical consequences for "resource-sharing" models of the dual-task bottleneck and will be considered in the Discussion section.

Figs. $6 \mathrm{C}$ and D illustrate T1 and T2 processing both at the sensor level and at the source level. The T1-evoked M450 was slower for slow RT1 compared to fast RT1 $(\mathrm{W}=6, \mathrm{p}<0.05)$, and similar in slow RT1 and blinked trials. Correspondingly, the delay observed on the T2evoked M550 during the PRP was increased for slow RT1 compared to fast RT1 (Fig. 6A) and the component was barely observable in blinked trials, as indicated by betas close to zero in Fig. 6D. These results suggest that, in agreement with our predictions, the central processing of $\mathrm{T} 2$ is delayed by $\mathrm{T} 1$ processing and can even fail if $\mathrm{T} 1$ processing is too slow. 
A Time after T2 onset

252-292 ms
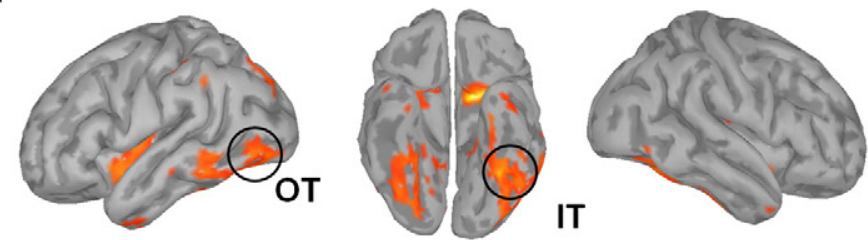

309-389 ms
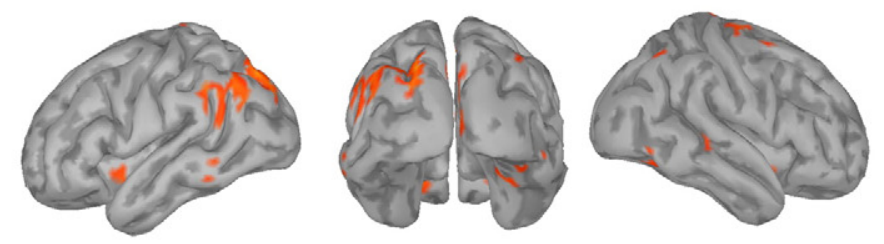

$390-470 \mathrm{~ms}$
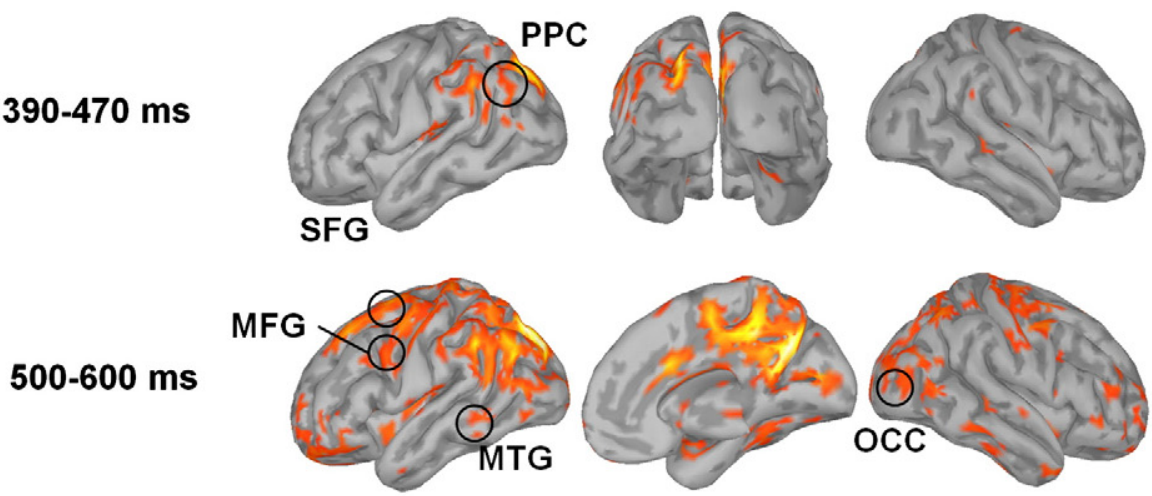

B
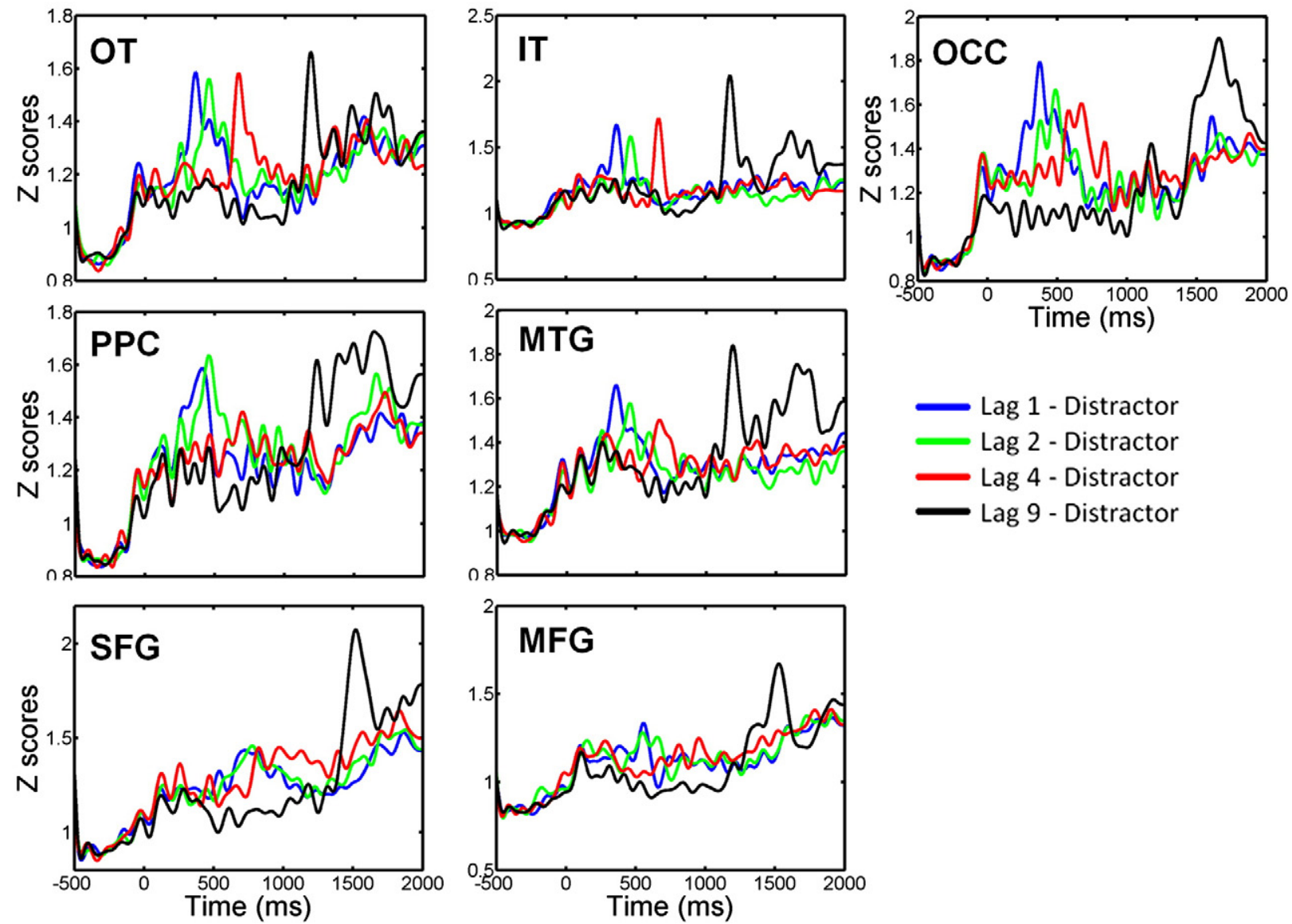

Lag 1 - Distractor

L Lag 2 - Distractor

L Lag 4 - Distractor

— Lag 9 - Distractor

Please cite this article as: Marti, S., et al., A shared cortical bottleneck underlying Attentional Blink and Psychological Refractory Period, NeuroImage (2011), doi:10.1016/j.neuroimage.2011.09.063 


\section{Discussion}

The present experiment shows that the PRP and the $\mathrm{AB}$ phenomena are deeply related at the brain level. We were able to obtain in a single experiment and with the very same stimuli both PRP trials and blinked trials (for a similar behavioral result, see (Wong, 2002)). We found that both kinds of trials underwent identical sensory processing but diverged during late central processing: activations in the frontal cortex were present but delayed during seen PRP trials, while they were sharply reduced in blinked trials. More importantly, we found a direct influence of task-1 duration on both the timing of frontal activations and the proportion of blinked trials. These results can be interpreted in a single theoretical framework if it is assumed that the "central" stages of task processing, which define the serial bottleneck, are precisely those available to conscious access (Dehaene et al., 2003; Marti et al., 2010). In a dual-task situation, conscious access to the second of two targets is not only pushed back in time (PRP), but it can even fail if $\mathrm{T} 1$ processing is too slow (AB).

By asking whether factors influencing the PRP would also influence $A B$, our study demonstrates the extended range of conditions under which $A B$ can be obtained. In past research, the $A B$ was typically explored within the visual modality while many PRP paradigms used distinct modalities of stimulation for T1 and T2. Here however, we obtained strong $\mathrm{AB}$ in a cross-modal PRP paradigm, thus confirming previous studies showing that an $A B$ can be found between modalities and suggesting that at least part of the phenomenon is due to an amodal, central limitation (Arnell, 2006; Arnell et al., 2004; Arnell and Larson, 2002; Jolicoeur, 1999a,b,c,d). Six of our subjects showed less than $10 \%$ of blinked trials at lag 1 (see the Method section). It is typical to observe a few such 'non-blinkers' participants in AB experiment (Martens et al., 2006), but the proportion of non-blinkers observed in the present study may seem larger than previously reported. The magnitude of the blink varies strongly across participants and across paradigms (Martens and Johnson, 2009; Martens et al., 2009; Martens et al., 2010a; Martens et al., 2010b; Martens et al., 2006; Martens and Valchev, 2009) and some studies did not find any cross modal AB (Duncan et al., 1997; Martens et al., 2010a; Potter et al., 1998). Thus, it is possible that there is a larger proportion of non-blinkers participants when using a cross-modal paradigm compared to standard visual paradigms, a topic for further research. In addition, the complexity of task 1 , which here was a simple two-choice response time task, might be an important factor influencing the proportion of non-blinkers (Martens et al., 2010b). Crucially, non-blinker participants are not immune to dual-task interference (Martens et al., 2010b), show a typically PRP delay, and thus do not constitute a violation of the present hypotheses. Furthermore, as detailed in the Results section, we verified that the results from the group of 18 participants and those from the 10 participants were comparable and we found the exact same effects and interactions, showing a significant $A B$, a significant PRP and importantly, a central role of RT1 speed in both phenomena.

An alternative explanation of our results would be that the crossmodal paradigm used here produced a task-switching effect between $\mathrm{T} 1$ and T2, and that such an effect is distinct from the $\mathrm{AB}$ (Potter et al., 1998). Given the difference between task 1 and 2 (i.e. tone discrimination versus letter identification), a task-switch was indeed required in our design. However, there is disagreement as to whether a different terminology should be used for paradigms involving task-switching or multiple modalities, compared to 'standard' $A B$ paradigm in which two visual masked targets are presented. Kawahara and colleagues
(2003) proposed that the general term "attentional blink" is suitable for both types of paradigms because they all share "a single critical factor - namely, a temporal delay between the onset of the second target and the time at which attention can be deployed to it" (Kawahara et al., 2003, p.350). We adopted this conclusion in the present research. In the context of our theoretical model (Zylberberg et al., 2010), both T1 processing and task-switching can potentially prolong the inattention period, thus resulting in a greater likelihood that T2 sensory information will have decayed and/or have been interrupted by a backward mask. Task-switching would then be sufficient (Kawahara et al., 2003) but not necessary to produce an $A B$. Indeed, there is evidence that cross-modal $\mathrm{AB}$ and lag 1 sparing can be found independently of taskswitching (Arnell and Larson, 2002; Ptito et al., 2008). However, in line with our model, task switching should be part of the central stage along with other processes such as conscious perception of $\mathrm{T} 1$ and decision making. Because of the serial property of the central stage, each process can contribute to the critical inattentional delay and, if the delay is long enough, to the $\mathrm{AB}$.

In addition, the absence of lag- 1 sparing in an $A B$ experiment might be considered as an index of a task-switching effect distinct from the AB (Potter et al., 1998). However, there is evidence that lag-1 sparing and the $\mathrm{AB}$ are two different phenomena. An interesting study examined the links between task-switching, lag 1 sparing and the $\mathrm{AB}$ (Peterson and Juola, 2000). The authors compared two conditions either involving or not a task-switch between T1 and T2. In both conditions, they found a virtually identical $A B$ pattern on $\mathrm{T} 2$ performance from lag 2 to lag 5 . The only difference was at lag 1 : no lag 1 sparing was found in the task-switch condition, making the decrease in T2 performance monotonic rather than U-shaped. Considering that our paradigm probably involved a task-switch, lag 1 sparing effect might have been overwhelmed by task-switching. It implies that $A B$ and lag 1 sparing are distinct phenomena and that task-switching affected lag 1 sparing but not the rest of the $A B$. In support to this view, there is evidence that the amplitude of $A B$ is unrelated to the amplitude of lag 1 sparing. In fact, the two phenomena appear to be statistically independent (Visser et al., 1999). One interpretation proposed by Visser et al. (1999) is that lag 1 sparing can occur only if there is a match between the task settings of T1 and T2. It would rely on sensory filters configured according to the current attentional set. If the same attentional set can be used for both $\mathrm{T} 1$ and $\mathrm{T} 2$, then $\mathrm{T} 2$ might benefit from this setting, resulting in an increase in task 2 performance. The $A B$, on the other hand, would occur later and would correspond to a delay in $\mathrm{T} 2$ processing because of the central bottleneck. From these evidences, we conclude that an $A B$ can be measured with or without lag 1 sparing depending on the paradigm used and, thus, that the absence of lag 1 sparing in our results does not mean an absence of $A B$.

It is well known in the PRP literature that the speed of RT1 has a strong influence on the speed of RT2. In fact, at short lag the two reaction times are strongly correlated (Pashler, 1994). We found here that the speed of RT1 also influenced the size of the blink only at short lag, confirming the results of a previous behavioural study (Jolicoeur, 1999d). This result show that when processing of task 1 is slow, task 2 processing is delayed and the risk of failing to consciously detect $\mathrm{T} 2$ increases. The central interference model can explain these results with the simple hypothesis that conscious access to a target stimulus is associated with the serial step of task processing and that the sensory buffer which is queued to be routed fades out in time.

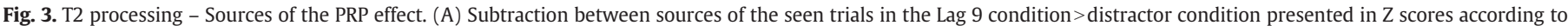

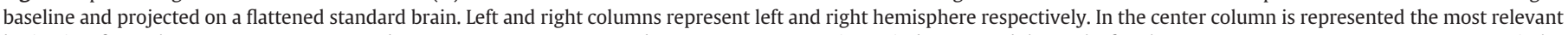

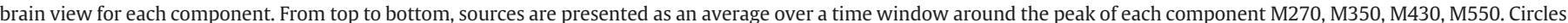

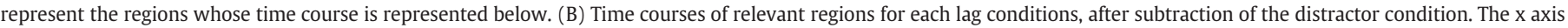

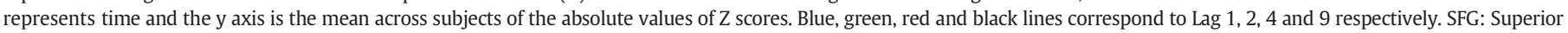

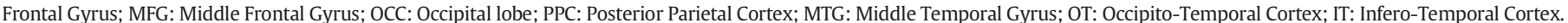


A

Long grad. Lat grad. Mag.

$353 \mathrm{~ms}$
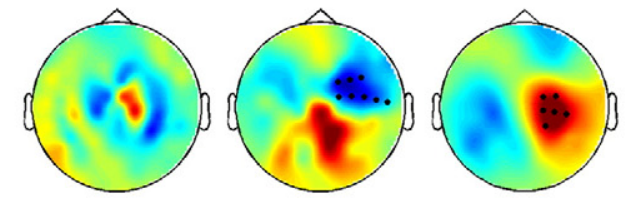

$420 \mathrm{~ms}$
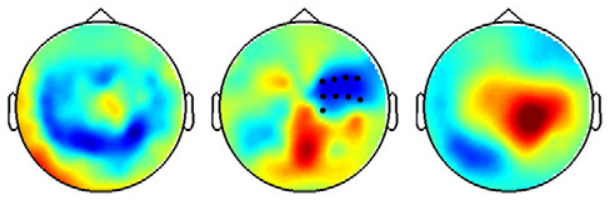

$720 \mathrm{~ms}$
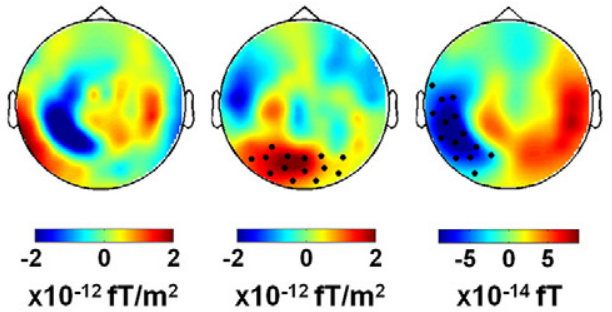

$\mathrm{x} 10^{-14} \mathrm{fT}$
B M270 Seen - Blinked

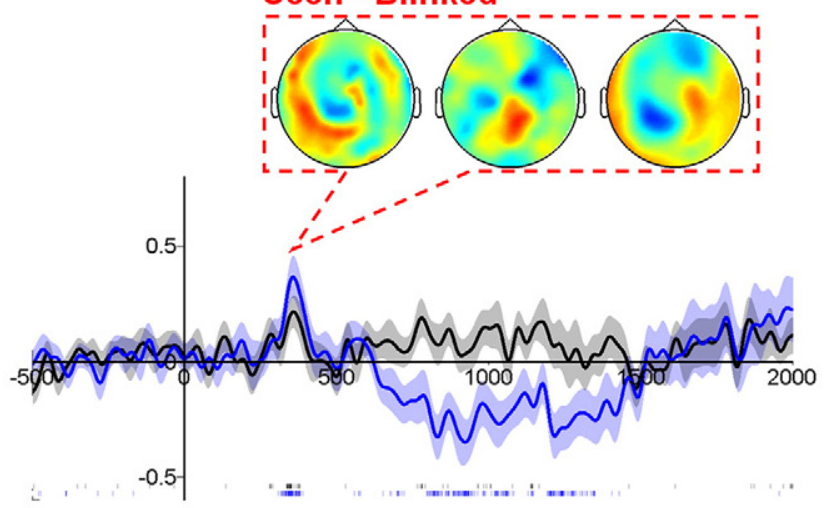

Blinked Lag 1 - Distractor

Long grad. Lat grad. Mag.
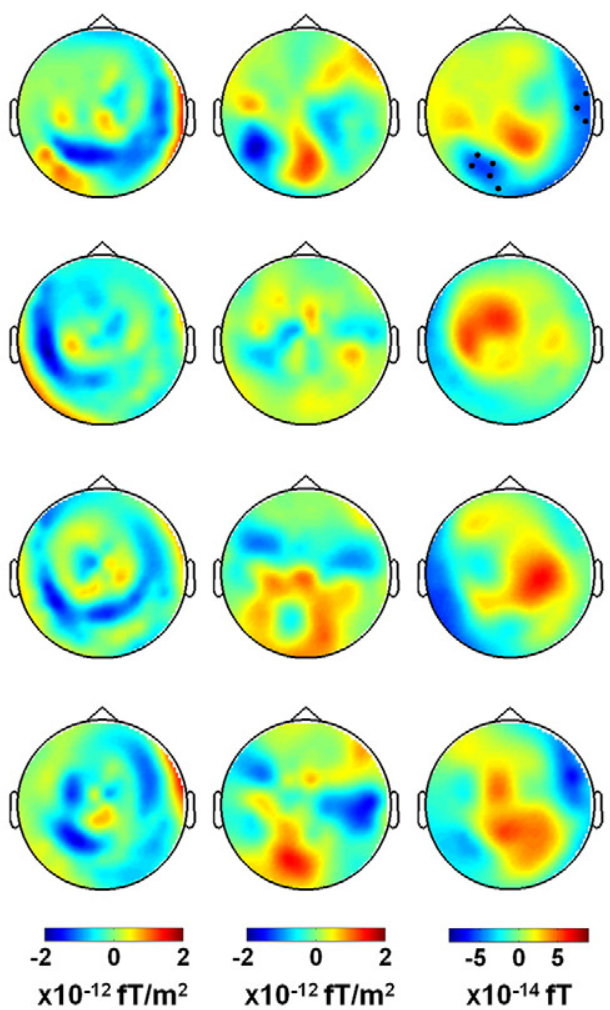

M350 Seen - Blinked
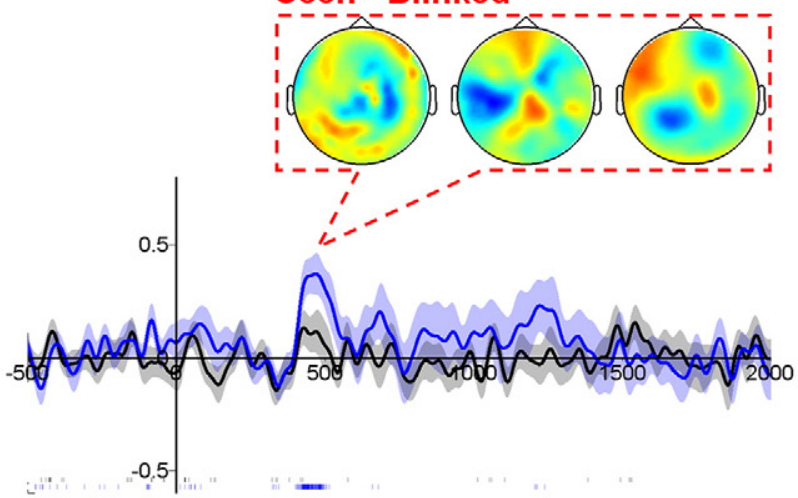

Seen

Blinked

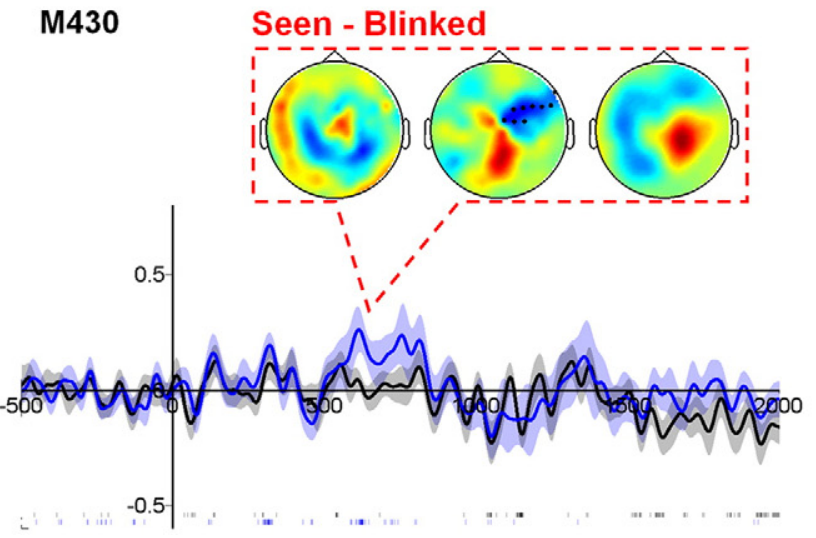

M550

Seen - Blinked

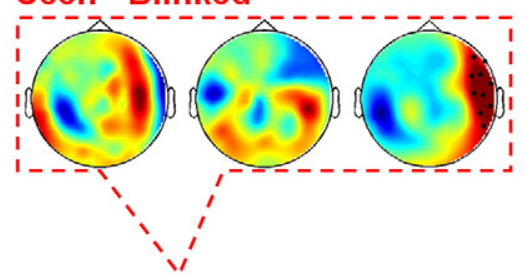

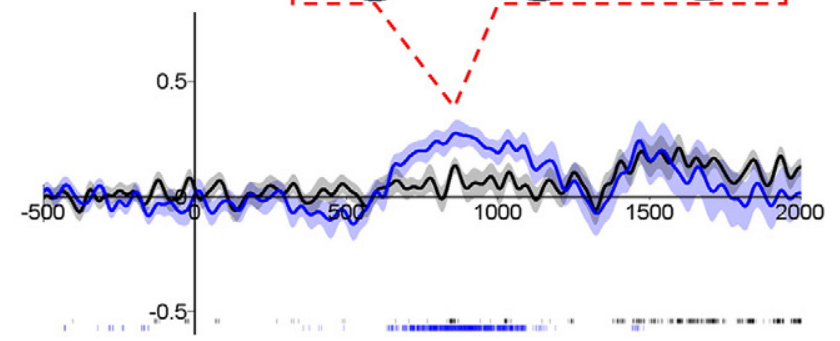




\section{Central processing in dual-tasks}

There is growing evidence that conscious access to a stimulus is systematically reflected in the P3 component of the ERPs (Del Cul et al., 2007; Donchin and Coles, 1988; Sergent et al., 2005). Previous studies examined separately the AB and the PRP and in both cases, reported that the P3 component of the ERPs is delayed at short compared to long inter target lags (Dell'acqua et al., 2005; Ptito et al., 2008; Sergent et al., 2005; Sessa et al., 2007; Sigman and Dehaene, 2008; Vogel and Luck, 2002). Here, for the first time, we directly compared, in the same paradigm, blinked trials and PRP trials at the brain level. We found that late magnetic components (i.e. the M430 and the M550 components) were the only ones to show a significant difference between seen and blinked trials, which suggests that they are related to the conscious access to $\mathrm{T} 2$. Second, the very same components were the only ones to be significantly delayed at short inter target lags, and to be significantly influenced by the speed of RT1. Thus, our study builds on previous results from PRP and $A B$ experiments and extends them by showing that the two phenomena arise from the same late components.

Previous studies in our lab have described conscious access as the entry of a stimulus in a global neuronal workspace (Dehaene and Naccache, 2001). Sensory information is processed in parallel and if the activity reaches a certain intensity threshold, it triggers the ignition of a network of brain areas including frontal, parietal and cingulate cortices (Dehaene et al., 2006; Del Cul et al., 2007; Kouider and Dehaene, 2007; Sergent et al., 2005; Sergent and Dehaene, 2004). We propose that brain activations observed mainly in the frontal cortex but also in the parietal cortex between $400 \mathrm{~ms}$ and $600 \mathrm{~ms}$ (the M430 and M550 components), correspond to the ignition of the workspace linked to the conscious access to T2. The present results suggest that processing a stimulus in the global workspace is part of a strictly serial process, which postpone the processing of any subsequent target. Evidences from other studies support this interpretation. For instance, it has been recently shown that, in order to trigger the $\mathrm{AB}$ at short T1-T2 lags, the minimum requirement is that subjects consciously perceive T1, even if they do not perform any further task on this target (Nieuwenstein et al., 2009a). Also, a recent behavioral PRP experiment conducted in our lab showed that, during the PRP, the conscious perception of T2, as estimated from participants' quantified introspection, was not time locked to the actual onset of $\mathrm{T} 2$, but to the end of task-1 processing (Marti et al., 2010). Our results establish that the serial dual-task bottleneck and conscious reportability are tightly linked phenomena. This interpretation does not exclude however the possibility that other cognitive operations over and above conscious access, such as task switching and task-2 setting, contribute to the serial bottleneck and to the observed late magnetic components.

\section{Target processing in a dual-task setting}

There is evidence in the $A B$ literature that, even if not seen, a stimulus can trigger deep sensory processing. For example, a blinked target word can trigger semantic priming over subsequent targets (Pesciarelli et al., 2007; Shapiro et al., 1997). At the brain level, early sensory components such as the N1 and P1 components are still present even if the target is blinked (Sergent et al., 2005). Blinked targets also evoke a late N400 component specific to word processing indicating that a blinked target can be processed up to the level of meaning (Luck et al., 1996; Sergent et al., 2005). In our study, we found that for all stimuli (i.e. seen targets, blinked targets and distractors) early sensory components up to $\sim 150$ ms were similar and unaffected by the dual-task interference. Furthermore, our recordings reveal an interesting finding: even during the blink, the brain continues to non-consciously separate the target letters from other distractor letters, although this distinction between targets and non-targets is arbitrarily defined by task instructions. Indeed, we found activations up to $270 \mathrm{~ms}$ that were specific to target stimuli, as opposed to distractors, yet remained detectable when the stimulus was not consciously perceived. Thus, even a blinked $\mathrm{T} 2$ still evoked a task-related activation. This surprising finding indicates that determining whether a letter is a T2 target continues to occur on blinked trials and proceeds in parallel with task 1 on PRP trials. Obviously, the set of target letters for task 2 (the letters Y and Z) must have been kept active throughout the trial, even during $\mathrm{T} 1$ processing.

Our findings are in line with previous results showing that highlevel cognitive processes, such as those underlying the N400 component, are not abolished either by the PRP or the AB (Vachon and Jolicoeur, 2011; Vogel et al., 1998). It also fits with evidence that both task-1 and task-2 sets are simultaneously maintained in a dual-task setting, as indicated for example by the fact that the response time to task 1 is typically slower in a dual-task versus a single-task setting, independently of the inter-target lag (Jiang et al., 2004; Sigman and Dehaene, 2005). Our result also fits with previous observations that attention can influence the processing of unconscious stimuli (Koch and Tsuchiya, 2007; Kouider and Dehaene, 2007). For instance, the N400 elicited by an unconscious word during a dual-task vanishes if task 1 has a high perceptual load (Giesbrecht et al., 2007). Another study showed that temporal attention modulated the priming effect of an unconscious stimulus (Naccache et al., 2002).

It is of course surprising that a T2 stimulus can be quickly classified as a task-relevant target, and yet remains undetected. This finding is, however, entirely compatible with the view that the limiting factor that ultimately determines the conscious perception of $\mathrm{T} 2$ is the availability of a parieto-frontal "global workspace" system (Dehaene and Naccache, 2001; Dehaene et al., 2003; Sergent and Dehaene, 2004). In our experiment, MEG activity revealed that this system is occupied for a considerable time by $\mathrm{T} 1$ processing and therefore cannot be immediately deployed for $\mathrm{T} 2$ perception, resulting in the $\mathrm{AB}$ phenomenon. During this period, the identified T2 target is thought to be in a "preconscious" state (Dehaene et al., 2006) - it is potentially accessible and reportable, but the actual moment of conscious access awaits central availability. An alternative view is tenable, according to which $\mathrm{T} 2$ is in fact already conscious, but this conscious state cannot be reported until the end of $\mathrm{T} 1$ processing, by which time it may have been forgotten and therefore blinked (Block, 2005; Lamme, 2006). While such a quick-forgetting interpretation of $A B$ is, by its very nature, almost irrefutable (Dennett, 1991), we note that it cannot explain one of our past findings: during the PRP, where subjects are conscious of $\mathrm{T} 2$, they still are blind to when $\mathrm{T} 2$ appeared, and misperceive it towards the end of T1 central processing (Marti et al., 2010). This observation does not fit easily with the idea that subjects were aware of T2 as soon as it appeared (but could not report it), but it fits well with the delayed conscious access predicted by the global workspace model.

\section{Other models of dual-task}

Alternative models of dual-task can explain part but not all the results we obtained in our experiment. For instance, a dominant

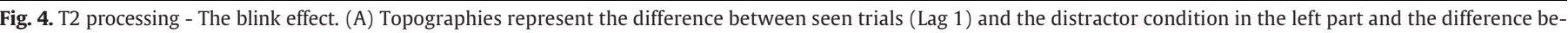

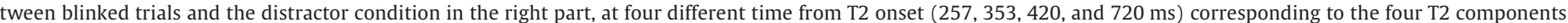

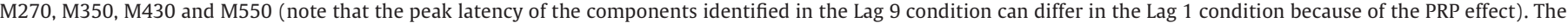

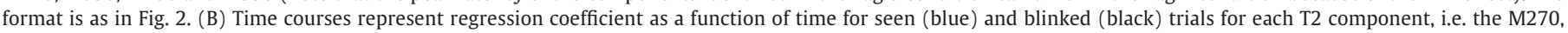
the M350, the M430 and the M550 (time samples are the same as in A). Shaded areas and bottom lines are as in Fig. 2. 
A

T2 Seen - Distractor

237-277 ms

313-393 ms

$380-460 \mathrm{~ms}$

670-770 ms

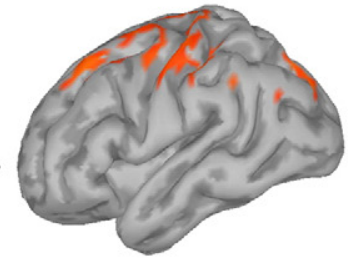

B
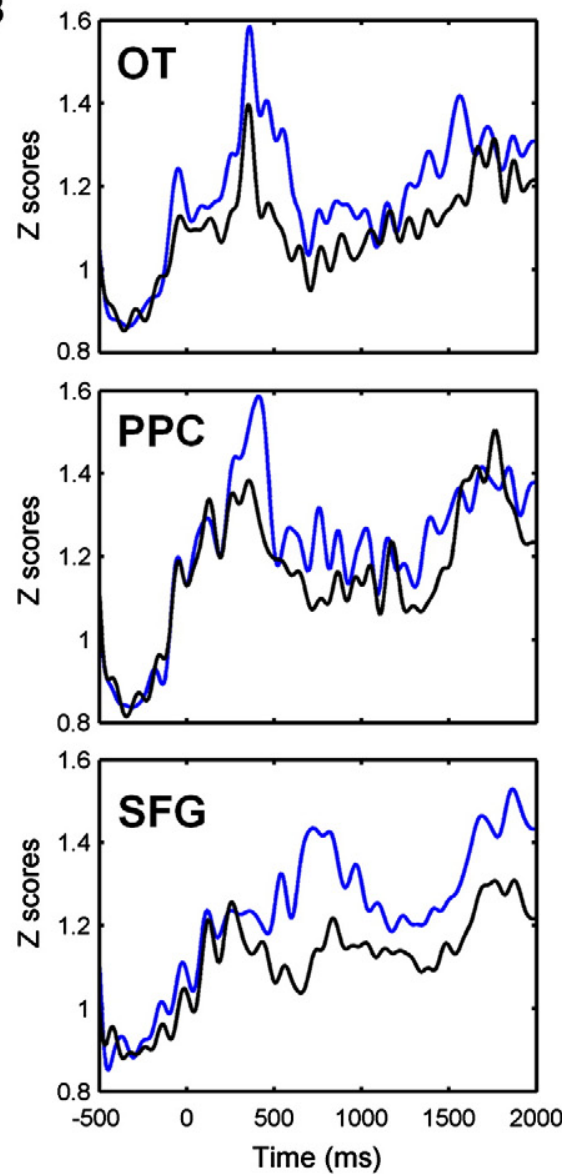

T2 Blinked - Distractor
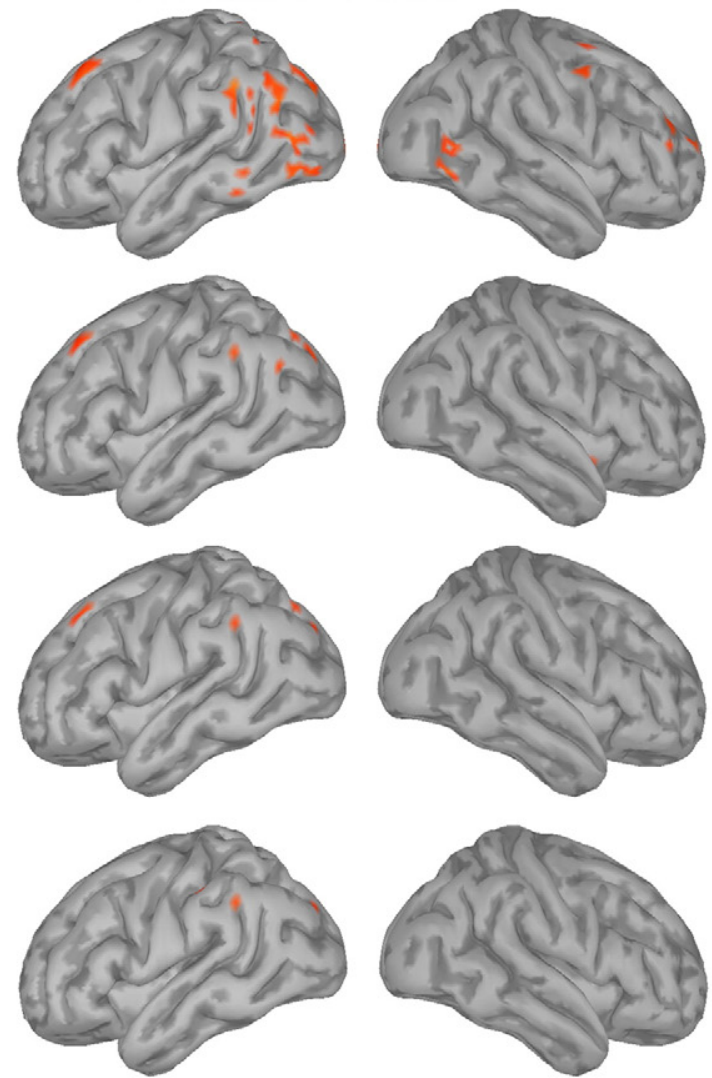
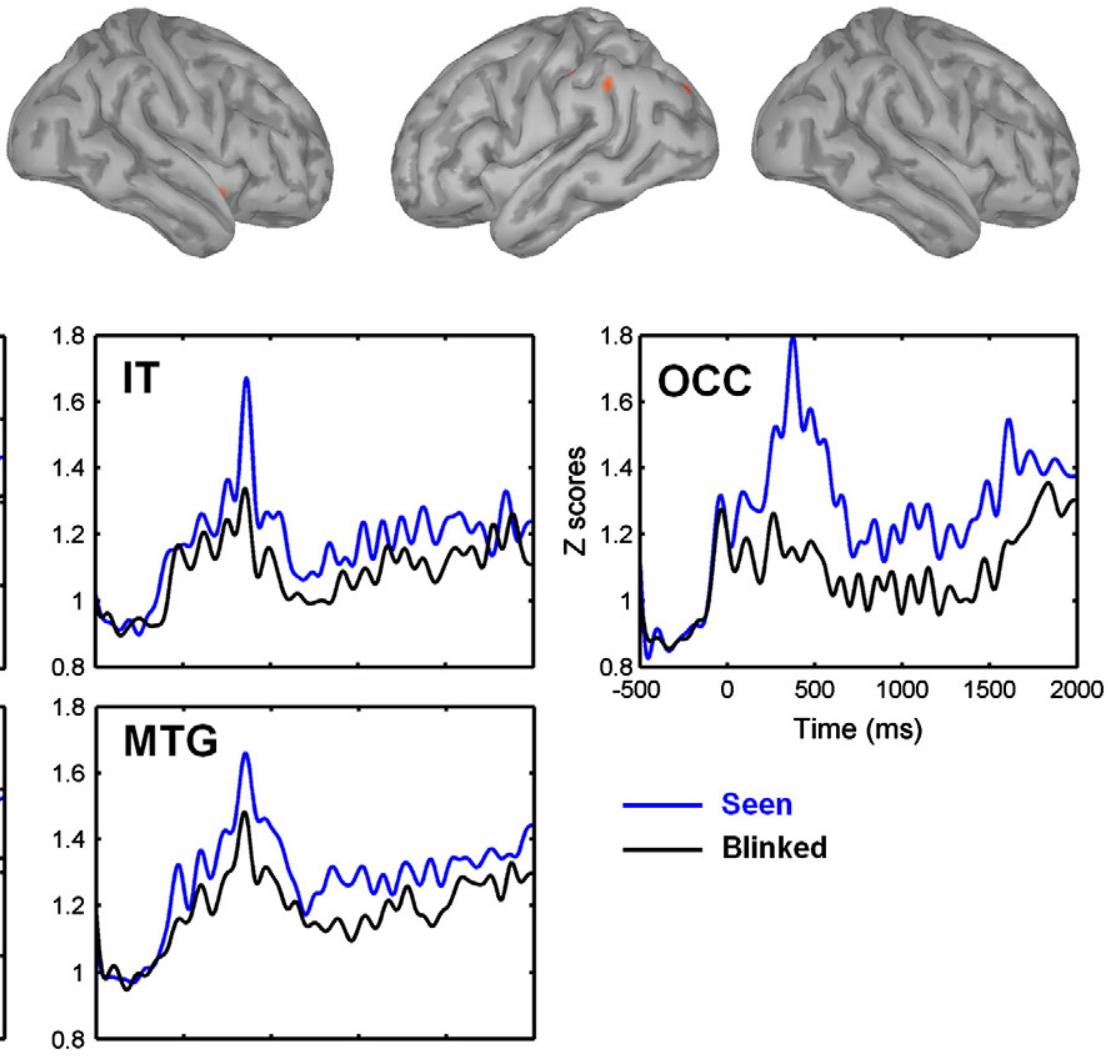

- Seen
Blinked

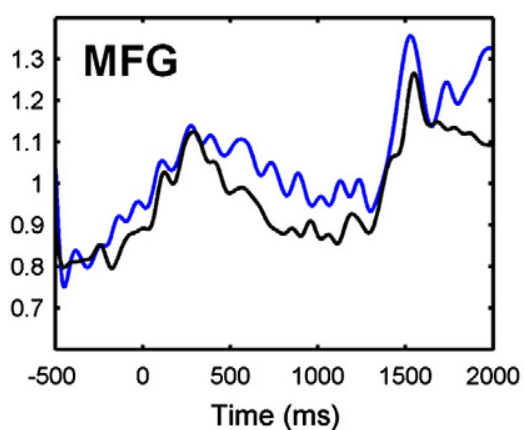

Please cite this article as: Marti, S., et al., A shared cortical bottleneck underlying Attentional Blink and Psychological Refractory Period, NeuroImage (2011), doi:10.1016/j.neuroimage.2011.09.063 
model of the attentional blink is the simultaneous type - serial token (ST2) model (Bowman and Wyble, 2007; Craston et al., 2009). The ST2 model shares several features of the central interference model. It also postulates two successive stages, first a parallel sensory stage and then a second stage of serial access to working memory and conscious perception. According to the ST2 model, a relevant item is first integrated at a sensory stage, and then temporally enhanced by attention allowing it to access working memory. In this model, during the interference period, the second stage is occupied encoding T1. During this period, T2 is therefore decaying in the sensory stage and may have vanished completely by the time the second stage is freed, an idea very similar to the one described in our model and neuronal simulation (Sigman and Dehaene, 2005, 2006, 2008; Zylberberg et al., 2010). A key difference, however, is that in the ST2 model, T2 is blinked because T1 processing precludes T2 from getting the necessary attentional enhancement. That is, the attentional task-related T2 enhancement is postponed until after T1 completion. This prediction is directly refuted by our present observation of enhanced activation in the visual cortex for targets relative to distractor stimuli, even on blinked trials, showing that target stimuli receive specific T2-locked processing relative to distractors. As noted above, although for simplicity our neuronal simulations (Zylberberg et al., 2010) also did not include any attentional modulation of non-conscious processing, its presence is not only compatible with the global-workspace framework, but was in fact one of its original predictions (Dehaene and Naccache, 2001; Naccache et al., 2002). Our findings suggest that blinked T2 benefit from attentional enhancement, but that this enhancement is not sufficient to allow T2 to access the global workspace because of T1 processing.

Other models of the $\mathrm{AB}$ proposed that the mask following $\mathrm{T} 1$ plays an essential role in the production of the $A B$, either because of a competition between targets and masks (Shapiro et al., 1994), because of a disruption of the current attentional set (Di Lollo et al., 2005) or because of distractor inhibition (Olivers and Meeter, 2008; Taatgen et al., 2009). However, these models can hardly explain (i) the influence of RT1 on T2 report and central components, as observed in the present results, and (ii) a strong $A B$ in our paradigm which used an unmasked sound as T1. In addition, there is evidence that a visual $\mathrm{AB}$ can be obtained even without masking (Kawahara et al., 2003; Nieuwenstein et al., 2009a; Nieuwenstein et al., 2009b) and that only conscious perception of $\mathrm{T} 1$ is required to produce the $\mathrm{AB}$ (Nieuwenstein et al., 2009a). These results suggest that the T1 mask contributes to the $A B$ but is not a prerequisite, which argues against models suggesting that the presence of a T1 mask triggers the $A B$. However, these results and ours are totally compatible with Chun \& Potter's bottleneck model (1995) which proposed that T1 processing monopolized a central stage which delays or prevents T2 of being consolidated.

Another model of dual-task proposes that central resources are shared between T1 and T2 processing (Shapiro et al., 2006; Tombu and Jolicoeur, 2003, 2005). In this model, central processing of T1 and T2 occur in parallel but the total amount of resources is shared between $\mathrm{T} 1$ and $\mathrm{T} 2$, which makes central processing of both tasks slower. As noted by its authors, the central resource sharing model assumes that resources can occasionally be allocated at $100 \%$ or $0 \%$ to one or the other task. Thus, this model encompasses the central interference model as a special case and, in this sense, is totally compatible with our observations. We note, however, that the hypothesis of resource sharing is not needed to account for our data. The specific prediction of the resource-sharing model is that during the interference period (i.e. at short lags), both $\mathrm{T} 1$ and $\mathrm{T} 2$ central components would remain time-locked to the onset of their respective stimuli, and that their duration would be extended. However, our results show that neither
RT1 nor T1 brain components were affected by the presentation of T2, as shown in the vast majority of PRP studies (Pashler, 1994; Sigman and Dehaene, 2008). More importantly, the T2-M430 and T2-M550 components were not wider in duration, but simply pushed back in time, compatible with an all-or-none effect of the T1 task (Sergent et al., 2005) rather than a partial sharing of resources.

The same argument stands against a computational model of the PRP which suggests that task 1 and task 2 are processed in parallel, but only the motor sequence for task 2 response is strategically withheld in working memory until task 1 is completed (Meyer and Kieras, 1997). Contrary to what we observed, this strategic response-deferment model (SRD) would predict wider T2-related central components instead of a delay effect. In addition, the model would also predict that components related to conscious perception of $\mathrm{T} 2$ would not suffer from dual-task interference. However, we found that components with amplitudes significantly stronger in seen trials compared to blinked trials were specifically delayed during the PRP. Hence our results argue against parallel conscious access to T1 and T2.

The model of executive control of visual attention (ECTVA) proposed by Logan \& Gordon (2001) suggest an essential role of strategy during the PRP. According to this model, an essential problem in dual-tasks would be to make the correct association between a stimulus and a response. In the context of ECTVA, one solution to this problem is to manipulate the priority of each task, making attentional processes of $\mathrm{T} 1$ and $\mathrm{T} 2$ strictly serial. The present data are compatible with ECTVA, although we note that this model does not make specific predictions regarding the attentional blink. One of the key differences between this model and ours is that we propose seriality as a property of conscious access, while ECTVA suggests that it is a strategy adopted by subjects. The two models differ in their predictions regarding training during dual-tasks. The ECTVA model predicts that if T1 and T2 are highly dissimilar, such as a sound and a letter, the serial effect should be weaker compared to highly similar stimuli, and might eventually completely vanish after training. Our model, on the other hand, predicts that training should not eliminate the PRP effect. A few studies examined training effect on the PRP but opposite results have been found (Hazeltine et al., 2002; Ruthruff et al., 2001; Schumacher et al., 2001; Van Selst et al., 1999). Recently, we revisited this issue by training subjects with over 10,000 trials in a PRP task (Kamienkowski et al., 2011). Careful mathematical analysis of response time distributions led to the conclusion that "extensive practice reduces the duration of central decision stages, but that the qualitative property of central seriality remains a structural invariant", a finding which is fully compatible with the present model and less compatible with the ECTVA model.

\section{Conscious perception in dual task}

According to the proposed model, even in a passive setting, conscious perception of a T1 stimulus should be sufficient to produce transient dualtask interference. Indeed, some studies have already observed dual-task interferences when no task was required for T1, the sole requirement being that $\mathrm{T} 1$ is attended and consciously perceived (Nieuwenstein et al., 2009a). Wong (2002) found a clear mixture of AB and PRP trials even when $\mathrm{T} 1$ was perceived passively. Interestingly, varying the intensity of T2 has opposite effects on the PRP and on the AB: decreasing T2 intensity augmented the probability of $\mathrm{AB}$, but had an underadditive effect on the PRP (a smaller impact on RT2 at short lag than at longer lag) and this effect was similar whether or not task 1 requires a speeded task (Jolicoeur et al., 2001; Wong, 2002). These results are easily understandable with the central interference model: the second target is processed at the sensory stage in parallel to $\mathrm{T} 1$ processing but decreasing stimulus intensity increases the duration of this stage and decreases

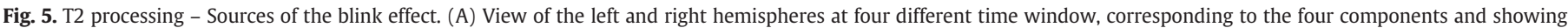

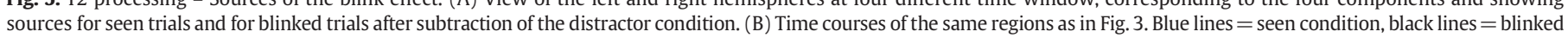
trials (both after subtraction of the distractor condition). 
A

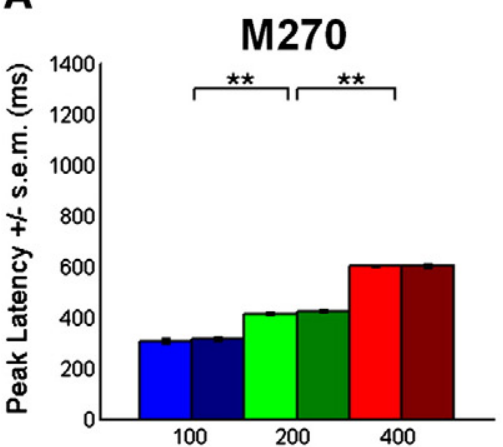

Inter target lag (ms)

B

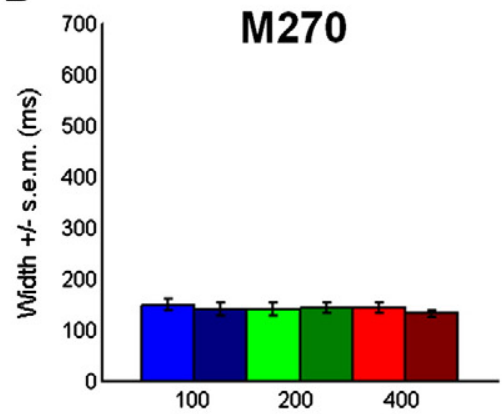

Inter target lag (ms)

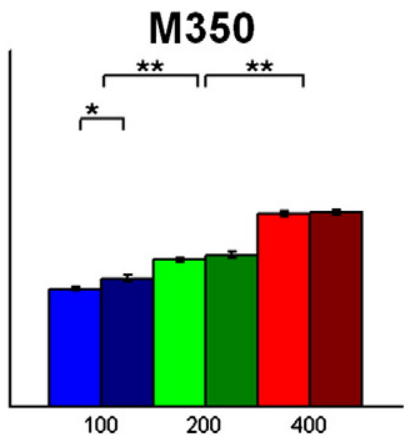

Inter target lag (ms)

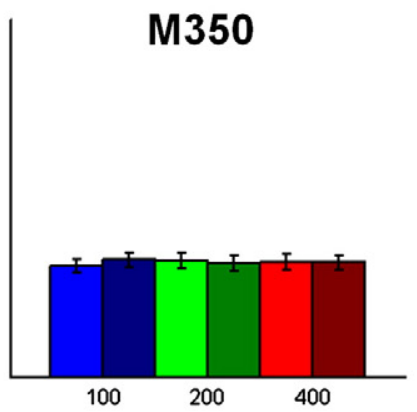

Inter target lag (ms)

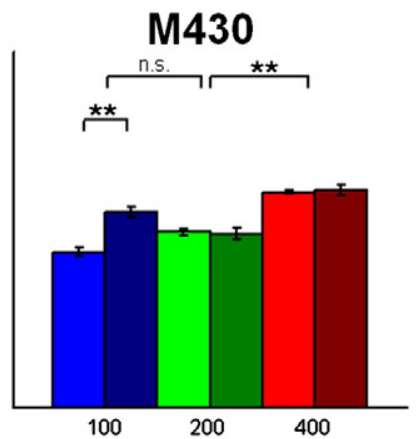

Inter target lag (ms)

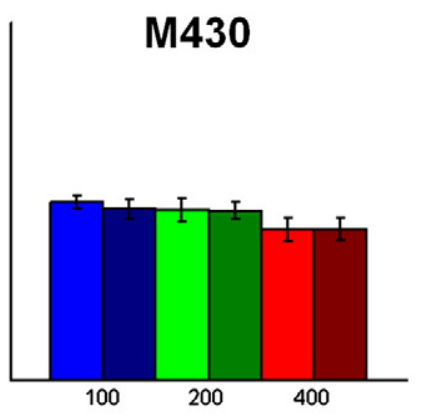

Inter target lag (ms)

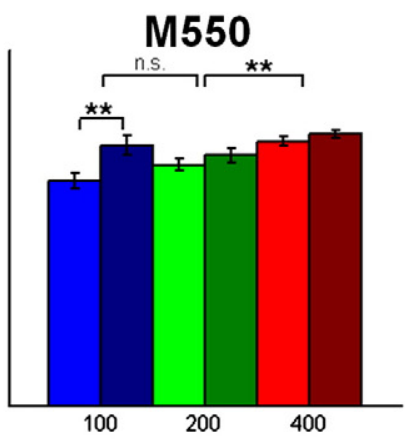

Inter target lag (ms)
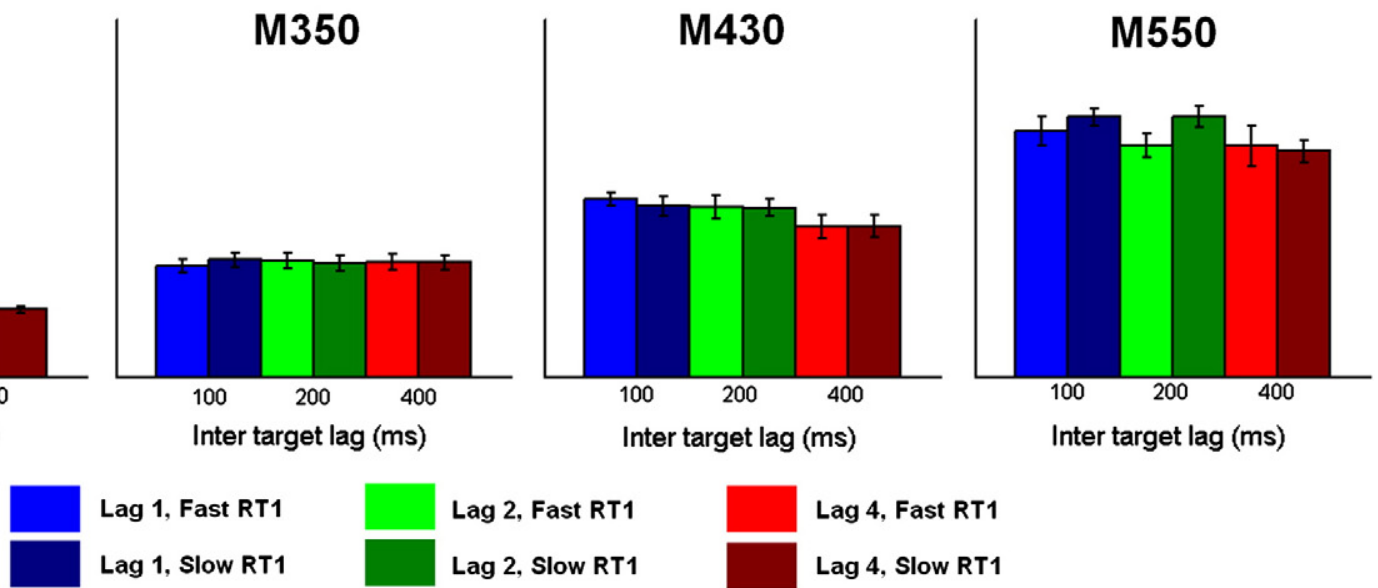

Inter target lag (ms)

Lag 4, Fast RT1

Lag 4, Slow RT1
D
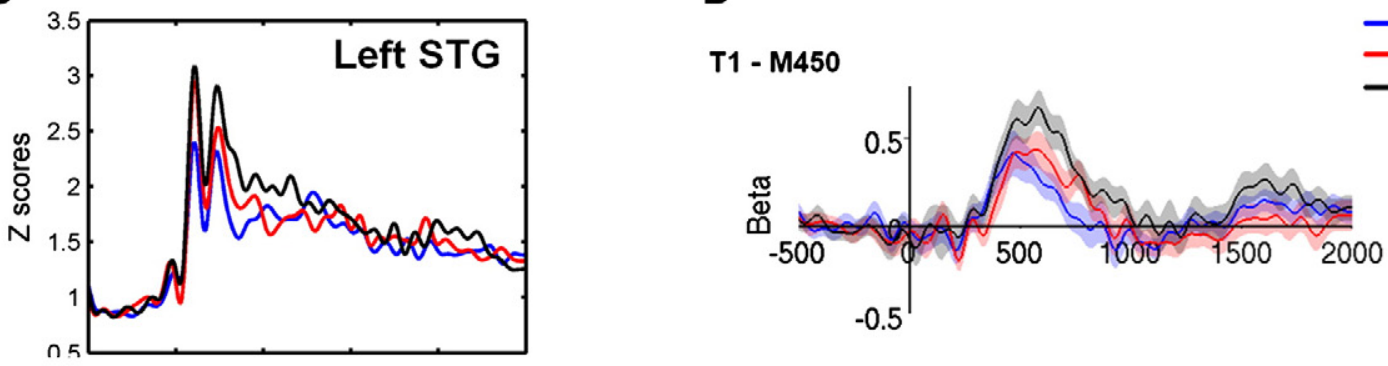

T2 - M550

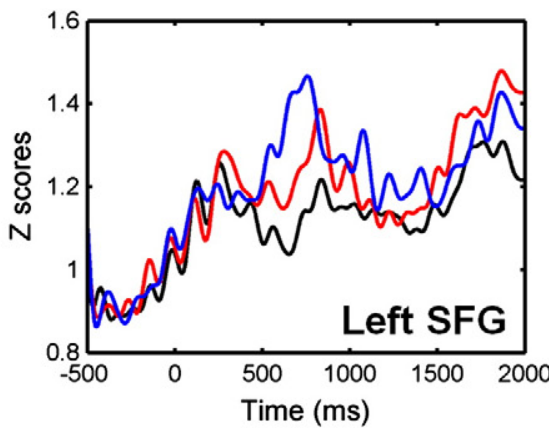

C

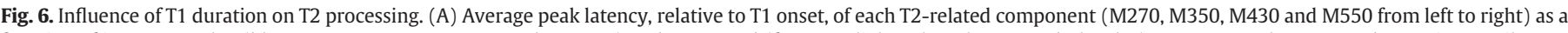

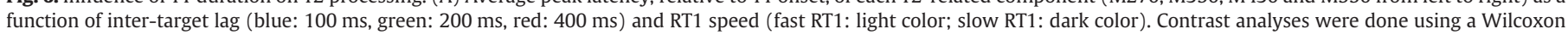

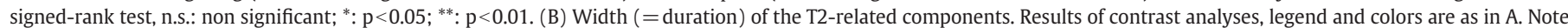

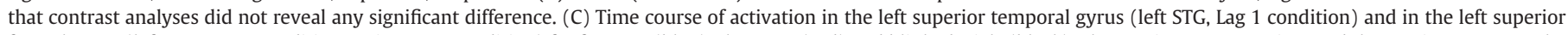

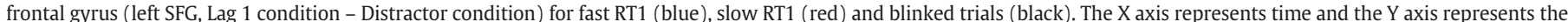

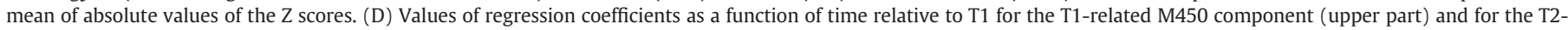

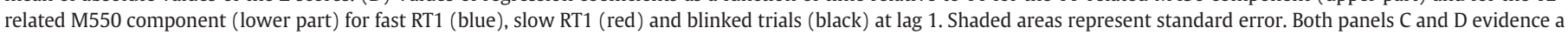
trade-off between the duration of $\mathrm{T} 1$ processing and the onset of $\mathrm{T} 2$ processing. 
the amount of sensory information. At short lag, the increased duration is absorbed by the PRP effect but less sensory information makes T2 more likely to be missed. Hence, these results again support the hypothesis that both the PRP and the AB arise from the same central serial stage which includes conscious perception.

\section{Conclusion}

Overall, our study shows that under dual-task circumstances, the conscious perception of the second target is pushed back in time and because of the evanescence of sensory information, conscious access can even fail, resulting in the attentional blink. The present demonstration that seriality is a prominent feature of conscious processing, although ancient (Posner and Snyder, 1975), bears considerable potential for future research. For instance, serial processing might serve as a proxy for the presence of conscious perception in patients unable to manifest a conscious report (see also Monti et al., 2010) or in non-human species in which subjective reports cannot be measured.

Supplementary materials related to this article can be found online at doi:10.1016/j.neuroimage.2011.09.063.

\section{Acknowledgments}

The project and the MEG equipment were supported by the Fondation pour la Recherche Médicale en France, the Bettencourt-Schueller foundation, the Région île-de-France, and the Human Frontier Science Program.

We are grateful to Virginie van Wassenhove, Etienne Labyt and François Tadel for help in data acquisition and analysis, and to Moti Salti and Aaron Schurger for constructive discussion.

\section{References}

Arnell, K.M., 2006. Visual, auditory, and cross-modality dual-task costs: electrophysiological evidence for an amodal bottleneck on working memory consolidation. Percept Psychophys. 68, 447-457.

Arnell, K.M., Helion, A.M., Hurdelbrink, J.A., Pasieka, B., 2004. Dissociating sources of dual-task interference using human electrophysiology. Psychon. Bull. Rev. 11, 77-83.

Arnell, K.M., Jenkins, R., 2004. Revisiting within-modality and cross-modality attentional blinks: effects of target-distractor similarity. Percept. Psychophys. 66, 1147-1161.

Arnell, K.M., Larson, J.M., 2002. Cross-modality attentional blinks without preparatory task-set switching. Psychon. Bull. Rev. 9, 497-506.

Becic, E., Dell, G.S., Bock, K., Garnsey, S.M., Kubose, T., Kramer, A.F., 2010. Driving impairs talking. Psychon. Bull. Rev. 17, 15-21.

Block, N., 2005. Two neural correlates of consciousness. Trends Cogn. Sci. 9, 46-52.

Bowman, H., Wyble, B., 2007. The simultaneous type, serial token model of tempora attention and working memory. Psychol. Rev. 114, 38-70.

Brisson, B., Jolicoeur, P., 2007a. Cross-modal multitasking processing deficits prior to the central bottleneck revealed by event-related potentials. Neuropsychologia 45 3038-3053.

Brisson, B., Jolicoeur, P., 2007b. A psychological refractory period in access to visual short-term memory and the deployment of visual-spatial attention: multitasking processing deficits revealed by event-related potentials. Psychophysiology 44 323-333.

Chun, M.M., Potter, M.C., 1995. A two-stage model for multiple target detection in rapid serial visual presentation. J. Exp. Psychol. Hum. Percept. Perform. 21, 109-127.

Craston, P., Wyble, B., Chennu, S., Bowman, H., 2009. The attentional blink reveals seria working memory encoding: evidence from virtual and human event-related potentials. J. Cogn. Neurosci. 21, 550-566.

Dehaene, S., Changeux, J.P., Naccache, L., Sackur, J., Sergent, C., 2006. Conscious, preconscious, and subliminal processing: a testable taxonomy. Trends Cogn. Sci. 10, 204-211.

Dehaene, S., Naccache, L., 2001. Towards a cognitive neuroscience of consciousness: basic evidence and a workspace framework. Cognition 79, 1-37.

Dehaene, S., Sergent, C., Changeux, J.P., 2003. A neuronal network model linking subjective reports and objective physiological data during conscious perception. Proc. Natl. Acad. Sci. U. S. A. $100,8520-8525$.

Del Cul, A., Baillet, S., Dehaene, S., 2007. Brain dynamics underlying the nonlinear threshold for access to consciousness. PLoS Biol. 5, e260.

Dell'Acqua, R., Jolicoeur, P., Pesciarelli, F., Job, C.R., Palomba, D., 2003. Electrophysiological evidence of visual encoding deficits in a cross-modal attentional blink paradigm. Psychophysiology 40, 629-639.

Dell'acqua, R., Jolicoeur, P., Vespignani, F., Toffanin, P., 2005. Central processing overlap modulates P3 latency. Exp. Brain Res. 165, 54-68.

Dell'Acqua, R., Turatto, M., Jolicoeur, P., 2001. Cross-modal attentional deficits in processing tactile stimulation. Percept. Psychophys. 63, 777-789.
Dennett, D., 1991. Consciousness explained. The Penguin Press, Allen Lane.

Di Lollo, V., Kawahara, J., Shahab Ghorashi, S.M., Enns, J.T., 2005. The attentional blink: resource depletion or temporary loss of control? Psychol. Res. 69, 191-200.

Donchin, E., Coles, M.G.H., 1988. Is the P300 Component a Manifestation of Context Updating. Behav. Brain Sci. 11, 357-374.

Duncan, J., Martens, S., Ward, R., 1997. Restricted attentional capacity within but not between sensory modalities. Nature $387,808-810$.

Duncan, J., Ward, R., Shapiro, K., 1994. Direct measurement of attentional dwell time in human vision. Nature 369, 313-315.

Dux, P.E., Ivanoff, J., Asplund, C.L., Marois, R., 2006. Isolation of a central bottleneck of information processing with time-resolved FMRI. Neuron 52, 1109-1120.

Giesbrecht, B., Sy, J.L., Elliott, J.C., 2007. Electrophysiological evidence for both perceptual and postperceptual selection during the attentional blink. J. Cogn. Neurosci. 19, 2005-2018.

Gold, J.I., Shadlen, M.N., 2001. Neural computations that underlie decisions about sensory stimuli. Trends Cogn. Sci. 5, 10-16.

Hazeltine, E., Teague, D., Ivry, R.B., 2002. Simultaneous dual-task performance reveals parallel response selection after practice. J. Exp. Psychol. Hum. Percept. Perform. 28, 527-545.

Hein, G., Parr, A., Duncan, J., 2006. Within-modality and cross-modality attentional blinks in a simple discrimination task. Percept. Psychophys. 68, 54-61.

Hillstrom, A.P., Shapiro, K.L., Spence, C., 2002. Attentional limitations in processing sequentially presented vibrotactile targets. Percept. Psychophys. 64, 1068-1082.

Jiang, Y., Saxe, R., Kanwisher, N., 2004. Functional magnetic resonance imaging provides new constraints on theories of the psychological refractory period. Psychol. Sci. 15, 390-396.

Jolicoeur, P., 1999a. Concurrent response selection demands modulate the attentional blink. J. Exp. Psychol. Hum. Percept. Perform. 25, 1097-1113.

Jolicoeur, P., 1999b. Dual-task interference and visual encoding. J. Exp. Psychol. Hum. Percept. Perform. 25, 596-616.

Jolicoeur, P., 1999c. Modulation of the attentional blink by on-line response selection: evidence from speeded and unspeeded task1 decisions. Mem. Cognit. 26, 1014-1032.

Jolicoeur, P., 1999d. Restricted attentional capacity between sensory modalities. Psychon. Bull. Rev. 6, 87-92.

Jolicoeur, P., Dell'acqua, R., Crebolder, J., 2000. Multitasking performance deficits: Forging links between the Attentional Blink and the Psychological Refractory Period. In: Monsell, S., Driver, J. (Eds.), Control of Cognitive Processes: Attention and Performance XVIII. The MIT Press, pp. 309-330.

Jolicoeur, P., Dell'acqua, R., Crebolder, J., 2001. The attentional blink bottleneck. In: Shapiro, $\mathrm{K}$. (Ed.), The limits of attention: Temporal constraints in human information processing. Oxford University Press, New York, NY, US, pp. 82-99.

Kamienkowski, J.E., Pashler, H., Dehaene, S., Sigman, M., 2011. Effects of practice on task architecture: combined evidence from interference experiments and randomwalk models of decision making. Cognition 119, 81-95.

Kawahara, J., Zuvic, S.M., Enns, J.T., Di Lollo, V., 2003. Task switching mediates the attentional blink even without backward masking. Percept. Psychophys. 65, 339-351.

Koch, C., Tsuchiya, N., 2007. Attention and consciousness: two distinct brain processes. Trends Cogn. Sci. 11, 16-22.

Kouider, S., Dehaene, S., 2007. Levels of processing during non-conscious perception: a critical review of visual masking. Philos. Trans. R. Soc. Lond. B Biol. Sci. 362, 857-875.

Lamme, V.A., 2006. Towards a true neural stance on consciousness. Trends Cogn. Sci. 10, 494-501.

Levy, J., Pashler, H., Boer, E., 2006. Central interference in driving: is there any stopping the psychological refractory period? Psychol. Sci. 17, 228-235.

Luck, S.J., Vogel, E.K., Shapiro, K.L., 1996. Word meanings can be accessed but not reported during the attentional blink. Nature 383, 616-618.

Martens, S., Johnson, A., 2009. Working memory capacity, intelligence, and the magnitude of the attentional blink revisited. Exp. Brain Res. 192, 43-52.

Martens, S., Johnson, A., Bolle, M., Borst, J., 2009. A quick visual mind can be a slow auditory mind. Individual differences in attentional selection across modalities. Exp. Psychol. $56,33-40$

Martens, S., Kandula, M., Duncan, J., 2010a. Restricted Attentional Capacity within but Not between Sensory Modalities: An Individual Differences Approach. PLoS One 5, e15280.

Martens, S., Korucuoglu, O., Smid, H.G., Nieuwenstein, M.R., 2010b. Quick minds slowed down: effects of rotation and stimulus category on the attentional blink. PLoS One 5, e13509.

Martens, S., Munneke, J., Smid, H., Johnson, A., 2006. Quick minds don't blink: electrophysiological correlates of individual differences in attentional selection. J. Cogn. Neurosci. $18,1423-1438$.

Martens, S., Valchev, N., 2009. Individual differences in the attentional blink. The important role of irrelevant information. Exp. Psychol. 56, 18-26.

Marti, S., Sackur, J., Sigman, M., Dehaene, S., 2010. Mapping introspection's blind spot: reconstruction of dual-task phenomenology using quantified introspection. Cognition $115,303-313$.

Meyer, D.E., Kieras, D.E., 1997. A computational theory of executive cognitive processes and multiple-task performance: Part 1. Basic mechanisms. Psychol. Rev. 104, 3-65.

Monti, M.M., Vanhaudenhuyse, A., Coleman, M.R., Boly, M., Pickard, J.D., Tshibanda, L., Owen, A.M., Laureys, S., 2010. Willful modulation of brain activity in disorders of consciousness. N. Engl. J. Med. 362, 579-589.

Naccache, L., Blandin, E., Dehaene, S., 2002. Unconscious masked priming depends on temporal attention. Psychol. Sci. 13, 416-424. 
Nieuwenstein, M., Van der Burg, E., Theeuwes, J., Wyble, B., Potter, M., 2009a. Temporal constraints on conscious vision: on the ubiquitous nature of the attentional blink. J. Vis. 9 (18), 11-14

Nieuwenstein, M.R., Potter, M.C., Theeuwes, J., 2009b. Unmasking the attentional blink. J. Exp. Psychol. Hum. Percept. Perform. 35, 159-169.

Olivers, C.N., Meeter, M., 2008. A boost and bounce theory of temporal attention. Psychol. Rev. $115,836-863$.

Pashler, H., 1994. Dual-task interference in simple tasks: data and theory. Psychol. Bull. $116,220-244$

Pashler, H., Johnston, J.C., 1989. Chronometric evidence for central postponement in temporally overlapping tasks. QJEP 41A, 19-45.

Pesciarelli, F., Kutas, M., Dell'acqua, R., Peressotti, F., Job, R., Urbach, T.P., 2007. Semantic and repetition priming within the attentional blink: an event-related brain potential (ERP) investigation study. Biol. Psychol. 76, 21-30.

Peterson, M.S., Juola, J.F., 2000. Evidence for distinct attentional bottlenecks in attention switching and attentional blink tasks. J. Gen. Psychol. 127, 6-26.

Posner, M., Snyder, C.R., 1975. Attention and cognitive control. Laurence Erlbaum, Hillsdale New Jersey.

Potter, M.C., Chun, M.M., Banks, B.S., Muckenhoupt, M., 1998. Two attentional deficits in serial target search: the visual attentional blink and an amodal task-switch deficit. J. Exp. Psychol. Learn. Mem. Cogn. 24, 979-992.

Ptito, A., Arnell, K., Jolicoeur, P., Macleod, J., 2008. Intramodal and crossmodal processing delays in the attentional blink paradigm revealed by event-related potentials. Psychophysiology 45, 794-803.

Raymond, J.E., Shapiro, K.L., Arnell, K.M., 1992. Temporary suppression of visual processing in an RSVP task: an attentional blink? J. Exp. Psychol. Hum. Percept. Perform. 18, 849-860.

Ruthruff, E., Johnston, J.C., Van Selst, M., 2001. Why practice reduces dual-task interference. J. Exp. Psychol. Hum. Percept. Perform. 27, 3-21.

Schumacher, E.H., Seymour, T.L., Glass, J.M., Fencsik, D.E., Lauber, E.J., Kieras, D.E. Meyer, D.E., 2001. Virtually perfect time sharing in dual-task performance: uncorking the central cognitive bottleneck. Psychol. Sci. 12, 101-108.

Sergent, C., Baillet, S., Dehaene, S., 2005. Timing of the brain events underlying access to consciousness during the attentional blink. Nat. Neurosci. 8, 1391-1400.

Sergent, C., Dehaene, S., 2004. Is consciousness a gradual phenomenon? Evidence for an all-or-none bifurcation during the attentional blink. Psychol. Sci. 15, 720-728.

Sessa, P., Luria, R., Verleger, R., Dell'Acqua, R., 2007. P3 latency shifts in the attentional blink: further evidence for second target processing postponement. Brain Res. 1137, 131-139.

Shapiro, K., Schmitz, F., Martens, S., Hommel, B., Schnitzler, A., 2006. Resource sharing in the attentional blink. Neuroreport 17, 163-166.
Shapiro, K.L., Driver, J., Ward, R., Sorensen, R.E., 1997. Priming from the attentional blink: A failure to extract visual tokens but not visual types. Psychol. Sci. 8, 95-100.

Shapiro, K.L., Raymond, J.E., Arnell, K.M., 1994. Attention to visual pattern information produces the attentional blink in rapid serial visual presentation. J. Exp. Psychol. Hum. Percept. Perform. 20, 357-371.

Sigman, M., Dehaene, S., 2005. Parsing a cognitive task: a characterization of the mind's bottleneck. PLoS Biol. 3, e37.

Sigman, M., Dehaene, S., 2006. Dynamics of the central bottleneck: dual-task and task uncertainty. PLoS Biol. 4, e220.

Sigman, M., Dehaene, S., 2008. Brain mechanisms of serial and parallel processing during dual-task performance. J. Neurosci. 28, 7585-7598.

Taatgen, N.A., Juvina, I., Schipper, M., Borst, J.P., Martens, S., 2009. Too much control can hurt: a threaded cognition model of the attentional blink. Cogn. Psychol. 59, 1-29.

Taulu, S., Kajola, M., Simola, J., 2004. Suppression of interference and artifacts by the Signal Space Separation Method. Brain Topogr. 16, 269-275.

Tombu, M., Jolicoeur, P., 2003. A central capacity sharing model of dual-task performance. J. Exp. Psychol. Hum. Percept. Perform. 29, 3-18.

Tombu, M., Jolicoeur, P., 2005. Testing the predictions of the central capacity sharing model. J. Exp. Psychol. Hum. Percept. Perform. 31, 790-802.

Tremblay, S., Vachon, F., Jones, D.M., 2005. Attentional and perceptual sources of the auditory attentional blink. Percept. Psychophys. 67, 195-208.

Vachon, F., Jolicoeur, P., 2011. Impaired semantic processing during task-set switching: evidence from the N400 in rapid serial visual presentation. Psychophysiology 48 $102-111$.

Van Selst, M., Ruthruff, E., Johnston, J.C., 1999. Can practice eliminate the psychological refractory period effect? J. Exp. Psychol. Hum. Percept. Perform. 25 1268-1283.

Visser, T.A., Bischof, W.F., Di Lollo, V., 1999. Attentional switching in spatial and nonspatial domains: Evidence from the attentional blink. Psychol. Bull. 125, 458-469.

Vogel, E.K., Luck, S.J., 2002. Delayed working memory consolidation during the attentional blink. Psychon. Bull. Rev. 9, 739-743.

Vogel, E.K., Luck, S.J., Shapiro, K.L, 1998. Electrophysiological evidence for a postperceptual locus of suppression during the attentional blink. J. Exp. Psychol. Hum. Percept. Perform. 24, 1656-1674.

Wong, K., 2002. The relationship between attentional blink and the psychological refractory period. J. Exp. Psychol. Hum. Percept. Perform. 28, 54-71.

Zylberberg, A., Fernandez, D., Roelfsema, P., Dehaene, S., Sigman, M., 2010. The Brain's Router: A Cortical Network Model of Serial Processing in the Primate Brain. PLoS Comput. Biol. 6, 1-23. 